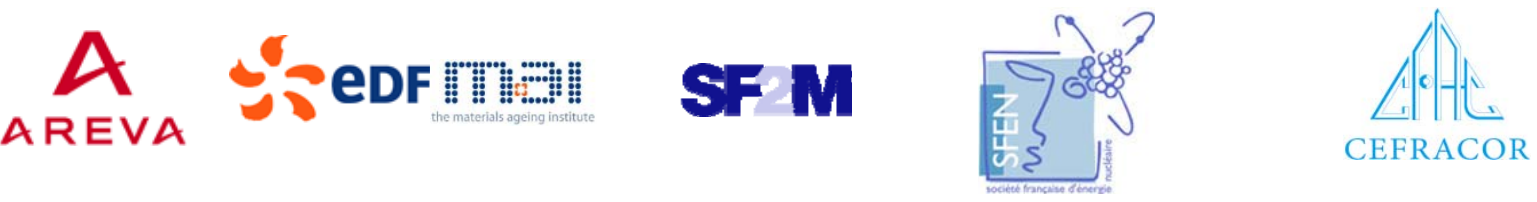

\section{MIN口S}

Centre of Excellence for Nuclear Materials

\section{Workshop}

Materials Innovation for Nuclear Optimized Systems

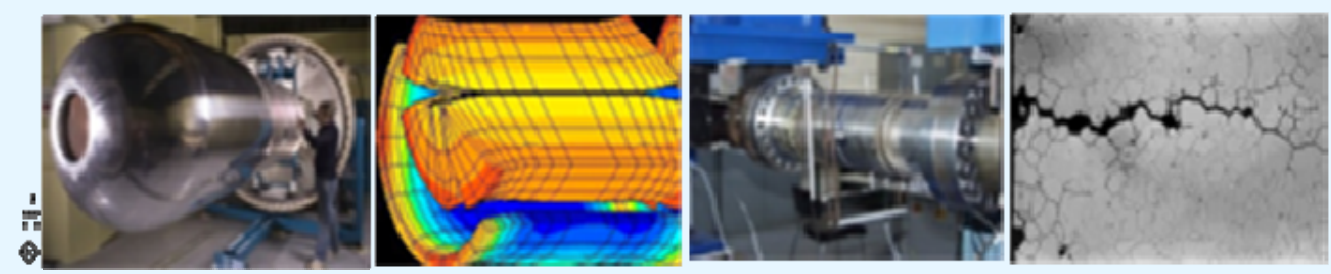

December 5-7, 2012, CEA - INSTN Saclay, France

\section{Christophe DOMAIN \\ EDF R\&D (France)}

Multiscale Modelling of Microstructure Evolution under Radiation Damage of Steels Based on Atomistic to Mesoscale Methods

Workshop organized by:

Christophe GALLÉ, CEA/MINOS, Saclay - christophe.galle@cea.fr Constantin MEIS, CEA/INSTN, Saclay - constantin.meis@cea.fr 


\title{
Multiscale Modelling of Microstructure Evolution under Radiation Damage of Steels Based on Atomistic to Mesoscale Methods
}

\author{
Christophe DOMAIN ${ }^{1,2}$ \\ ${ }^{1}$ EDF R\&D - MMC (Moret sur Loing, France) \\ ${ }^{2}$ EDF-CNRS joint laboratory EM2VM (Study and Modeling of the Microstructure for Ageing of Materials)
}

Structural metallic materials used in nuclear facilities are submitted to irradiation which induce the creation of large amounts of point defects, which leads to modifications of the microstructure and the mechanical properties. In nuclear power plants, the main structural materials are: the pressure vessel (ferritic steels), the internal structure (austenitic steels).

In order to simulate the microstructure evolution with the objective to predict it, multiscale modelling tools are developed (Fig. 1). For this purpose different simulation methods are used and developed in order to treat the different physical phenomena occurring at different time scales and length scales: ab initio, classical molecular dynamics, kinetic Monte Carlo, dislocation dynamics, phase field [1]. These simulations are very CPU demanding and take advantage of the development of High Performance Computing machines.

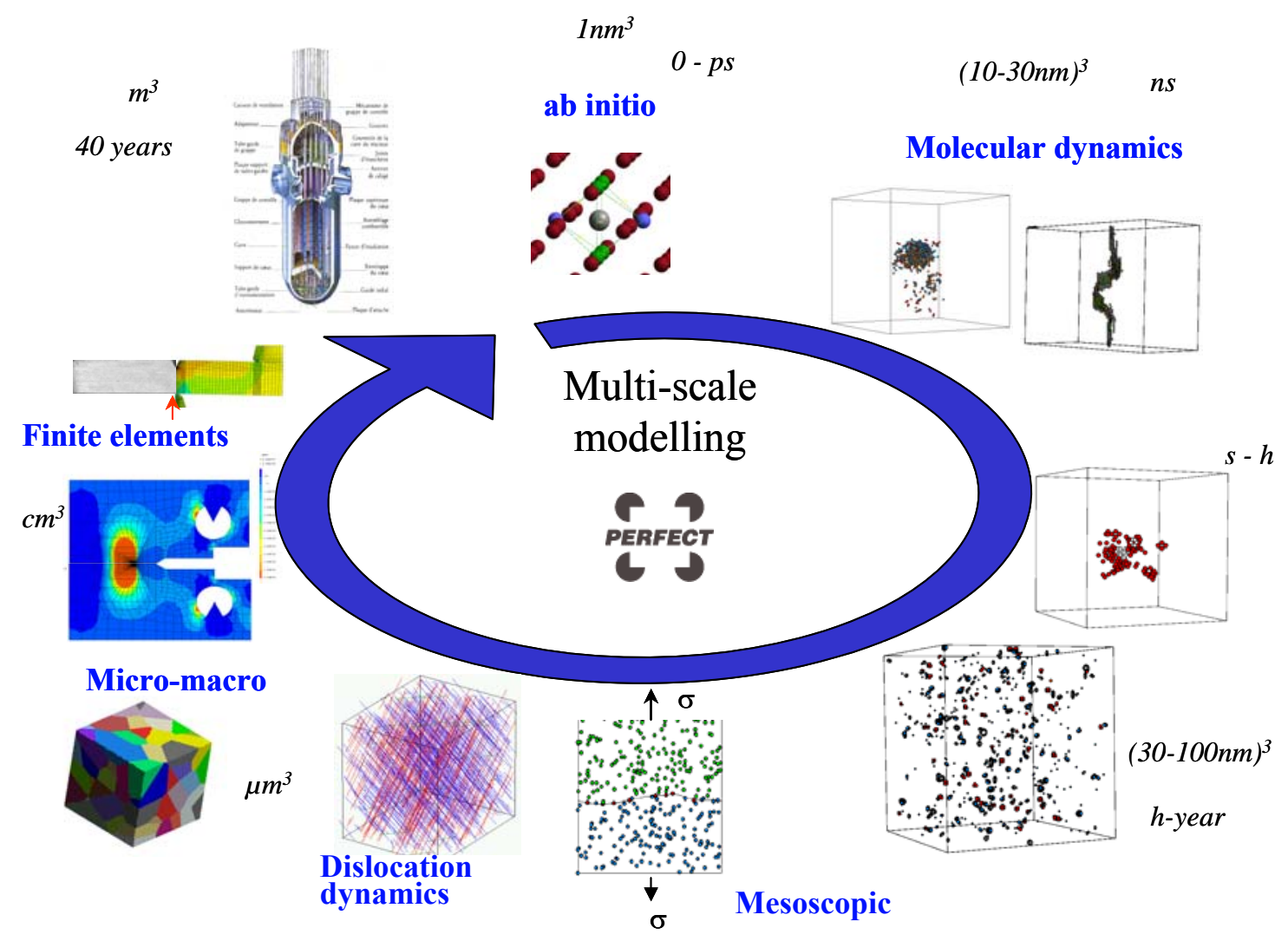




\section{Fig. 1: Multiscale modelling scheme applied within the PERFORM-60 project to the pressure vessel and internal material microstructure.}

The microstructure evolution under irradiation is obtained starting from the neutron spectrum to obtain the primary damage (displacement cascades), followed by the evolution of the point defects formed and their accumulation (Fig. 2).
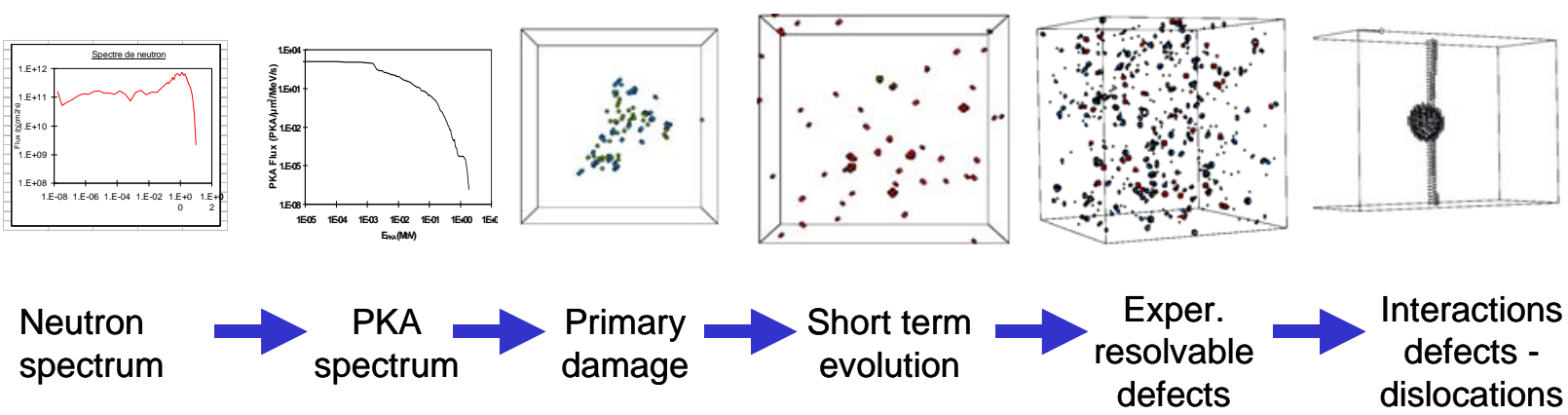

Fig. 2: Microstructure modelling under irradiation.

The point defects created (vacancy and self interstitials) under irradiation often interact with the solute elements present in the materials. Solutes can precipitate and/or segregate on point defect clusters (loops or voids) or extended defects (dislocations, grain boundaries). These modifications of the microstructure affect directly the mechanical properties of the materials. Thus, modelling should take into account the most important solute elements in the chemical composition of the industrial alloys.

For the pressure vessel steels (for which an important international efforts is done in particular thanks to the PERFECT [2] and PERFORM-60 european projects) the evolution of the microstructure of dilute $\mathrm{Fe}$ alloys as complex as Fe-CuNiMnSiP-C under irradiation are modelled using a multiscale approach based on ab initio, molecular dynamics and kinetic Monte Carlo (KMC) simulations. In these atomic KMC simulations, both self interstitials and vacancies, isolated or in clusters, as well as carbon atoms are modelled [3]. The short term evolution of the microstructure is simulated. The medium to long term evolution of the microstructure is obtained by object KMC and cluster dynamics, considering a "grey" material. The interaction of some of these defects with dislocations are characterised by molecular dynamics in order to be used in mesoscopic dislocation dynamics. A similar approach is developed for austenitic materials modelled by a concentrated FeCrNi alloy $(\gamma$ $\mathrm{Fe}_{70} \mathrm{Cr}_{20} \mathrm{Ni}_{10}$ ). The thermal ageing (without irradiation) of $\mathrm{FeCr}$ alloys will also be presented.

In the framework of the european projects dedicated to the pressure vessel steels and the austenitic steels, the multiscale modelling methods of the microstructure have been capitalised within two tools (RPV and INTERN) [4].

\section{References}

[1] C. S. Becquart, C. Domain, Metallurgical and Materials Transactions A 42 (2011) 852.

[2] Spetial Issue: PERFECT project. Journal of Nuclear Materials, 406 (2010).

[3] C.S. Becquart, C. Domain, Phys. Status Solidi B 247 (2010) 9.

[4] G. Adjanor et al., J. Nucl. Mater 406 (2010) 175. 
Workshop Materials Innovation for Nuclear Optimized Systems MINOS, Saclay, Dec 2012

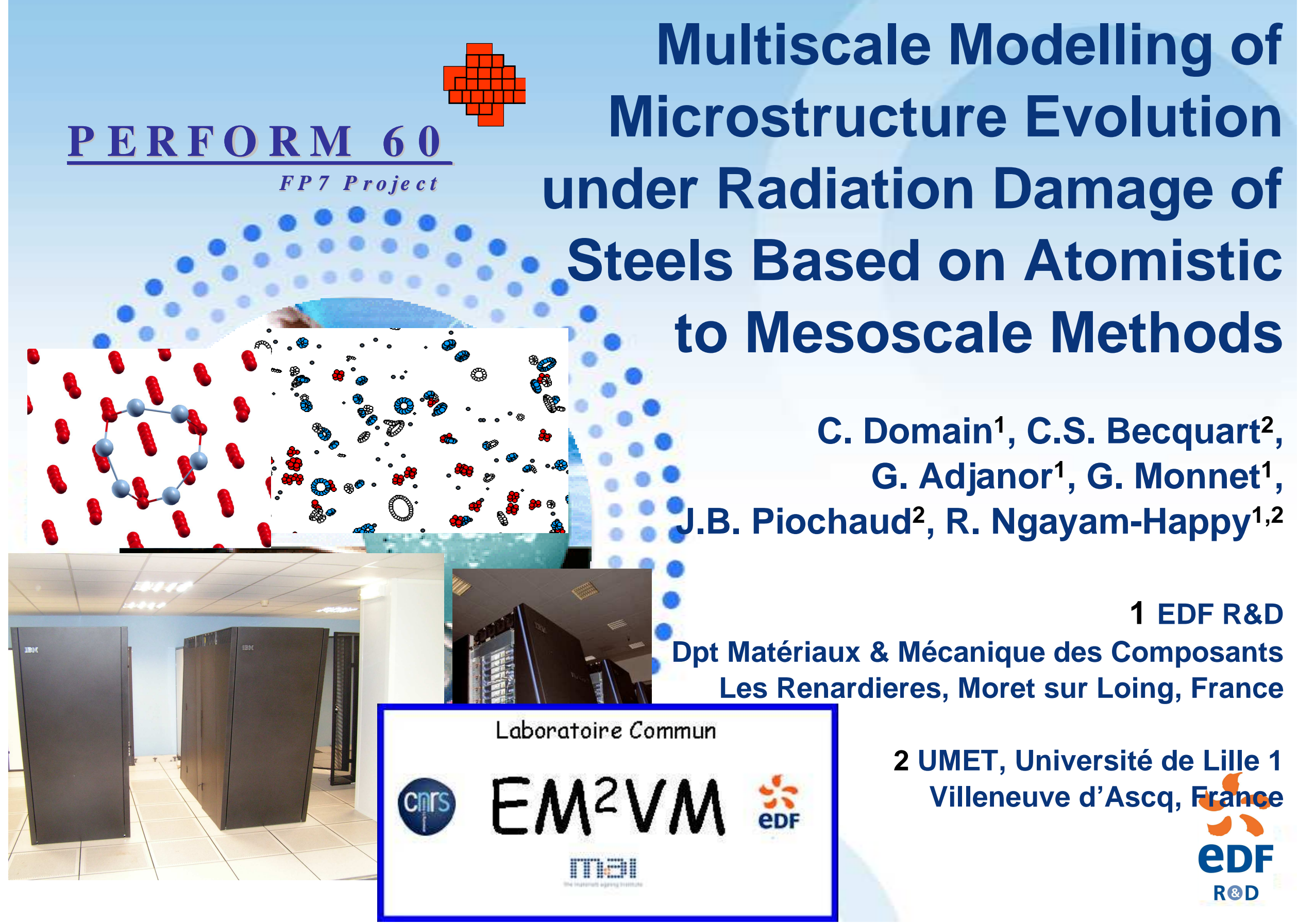




\section{Lifetime extension: Materials ageing prediction}

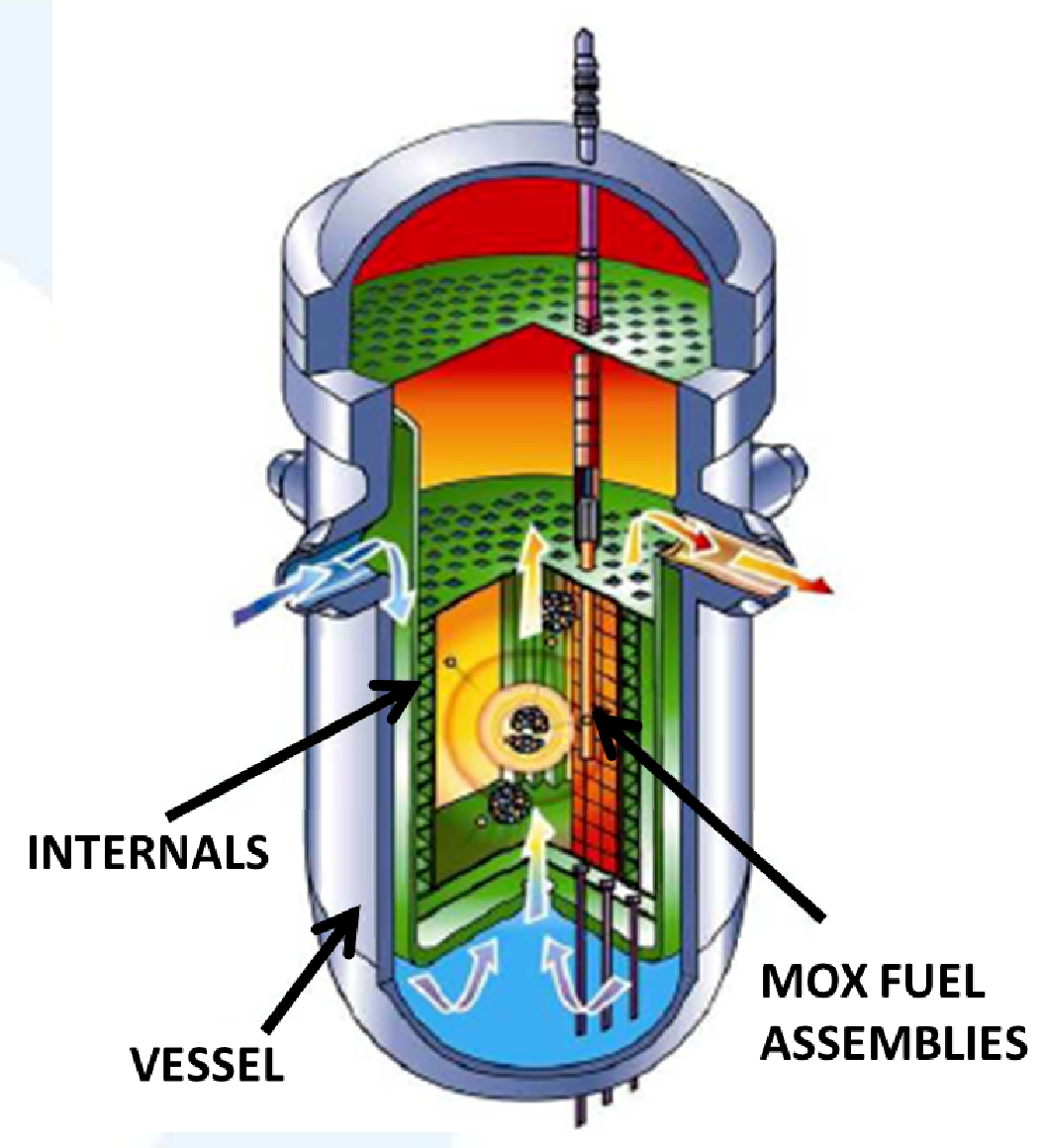

- To improve quantitative predictions of ageing of irradiated structural materials in nuclear power plants in order to gain margins.

- Challenge: to predict the evolution of hundred of tons over more than 40 years based on physical phenomena occurring at the nanometer scale and picosecond times $\left(10^{-12} \mathrm{~s}\right)$

- Construction and improvement of multiscale modelling methods allowing to better take into account the material composition and radiation damage

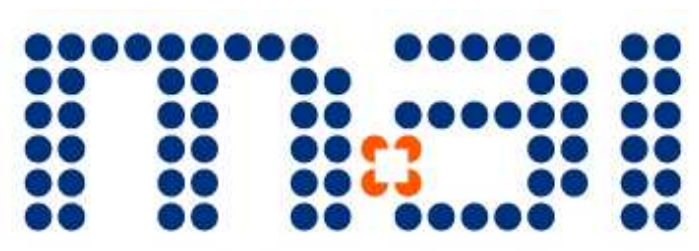




\section{Microstructure evolution of $\mathrm{Fe}$ alloys under irradiation}

\section{RPV Fe ferritic alloys}

- microstructure modelling short \& long term evolution

- plasticity

Si P Mn Ni Cu

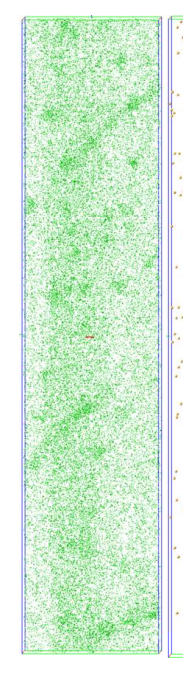

$30 \times 30 \times 140 \mathrm{~nm}^{3}$

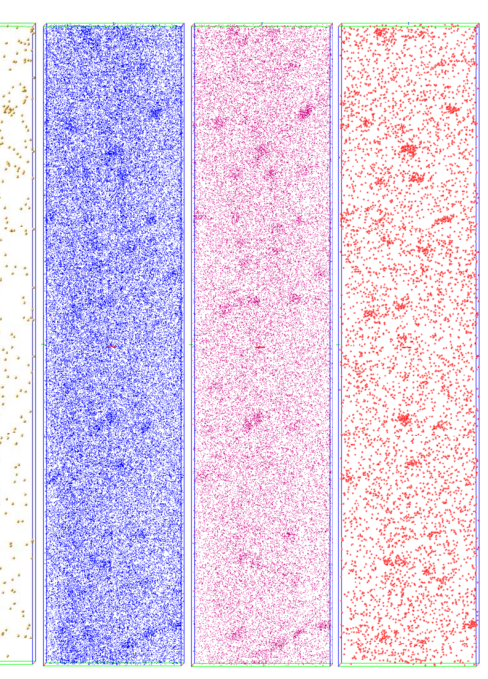

$\mathrm{Si}+\mathrm{P}+\mathrm{Mn}+\mathrm{Ni}+\mathrm{Cu}$

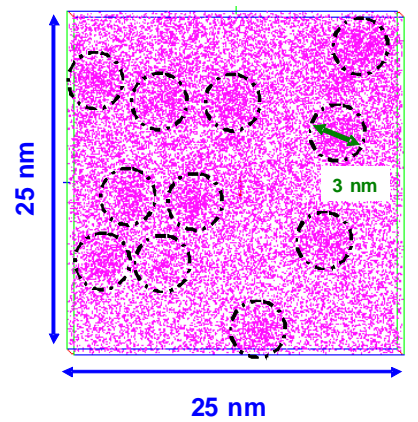

[H. Huang PhD

SAT@GPM Rouen]

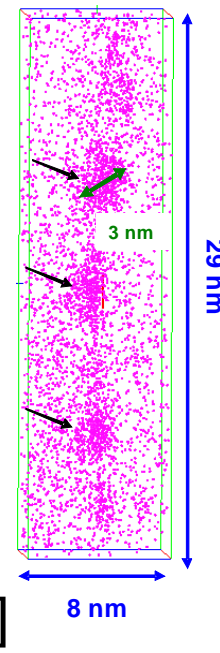

\section{Austenitic alloys}

- FeNiCr AKMC RIS modelling

[A. Volgin PhD SAT@GPM Rouen ]

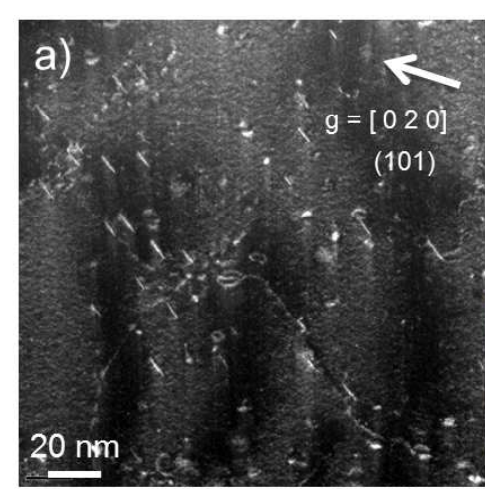

c)
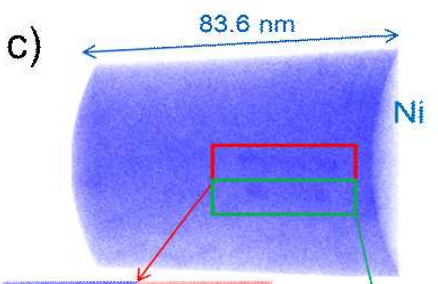

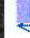

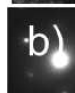

b)
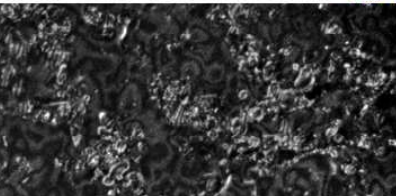

(3)

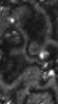

1.. 8.2. 


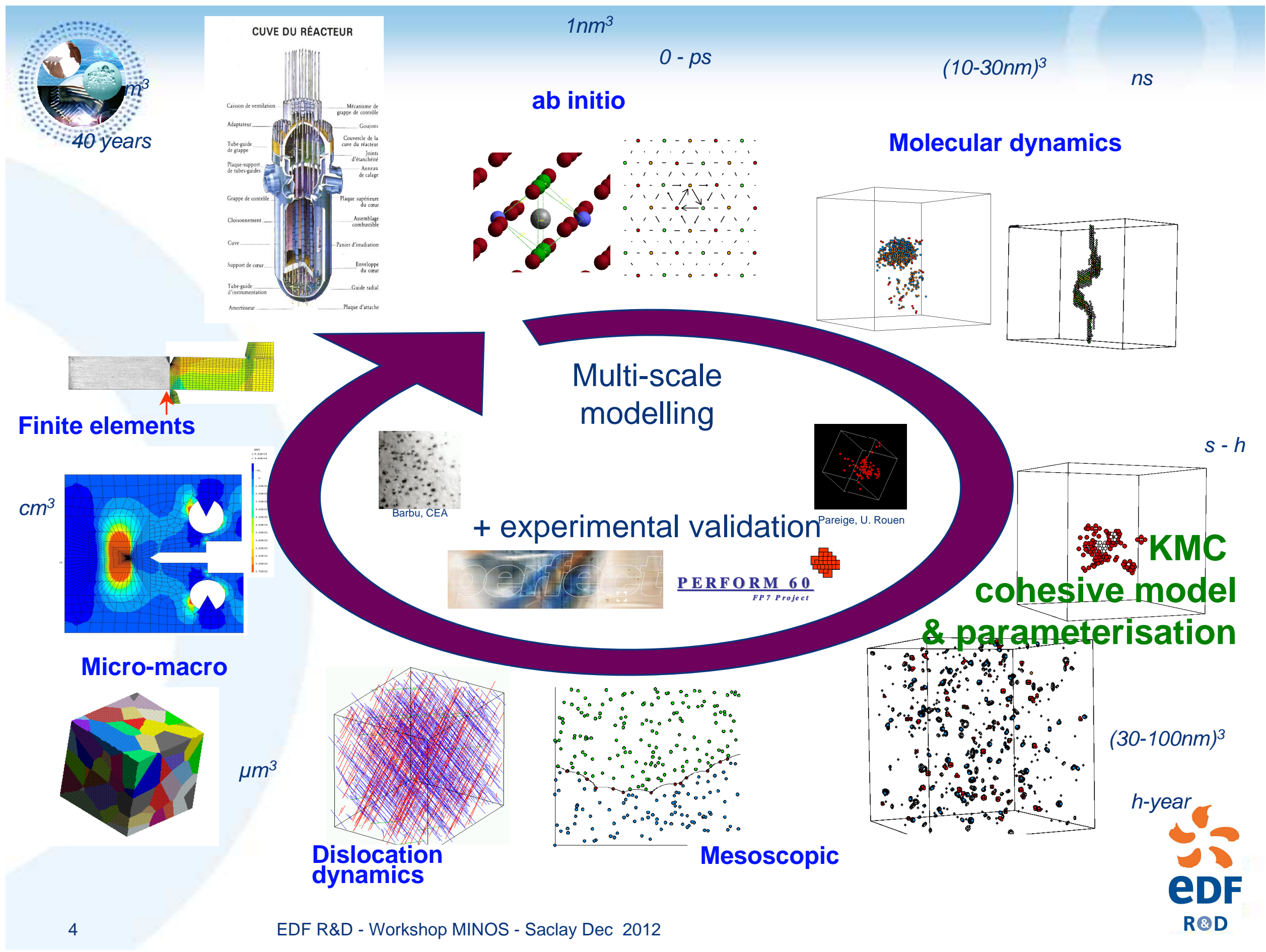




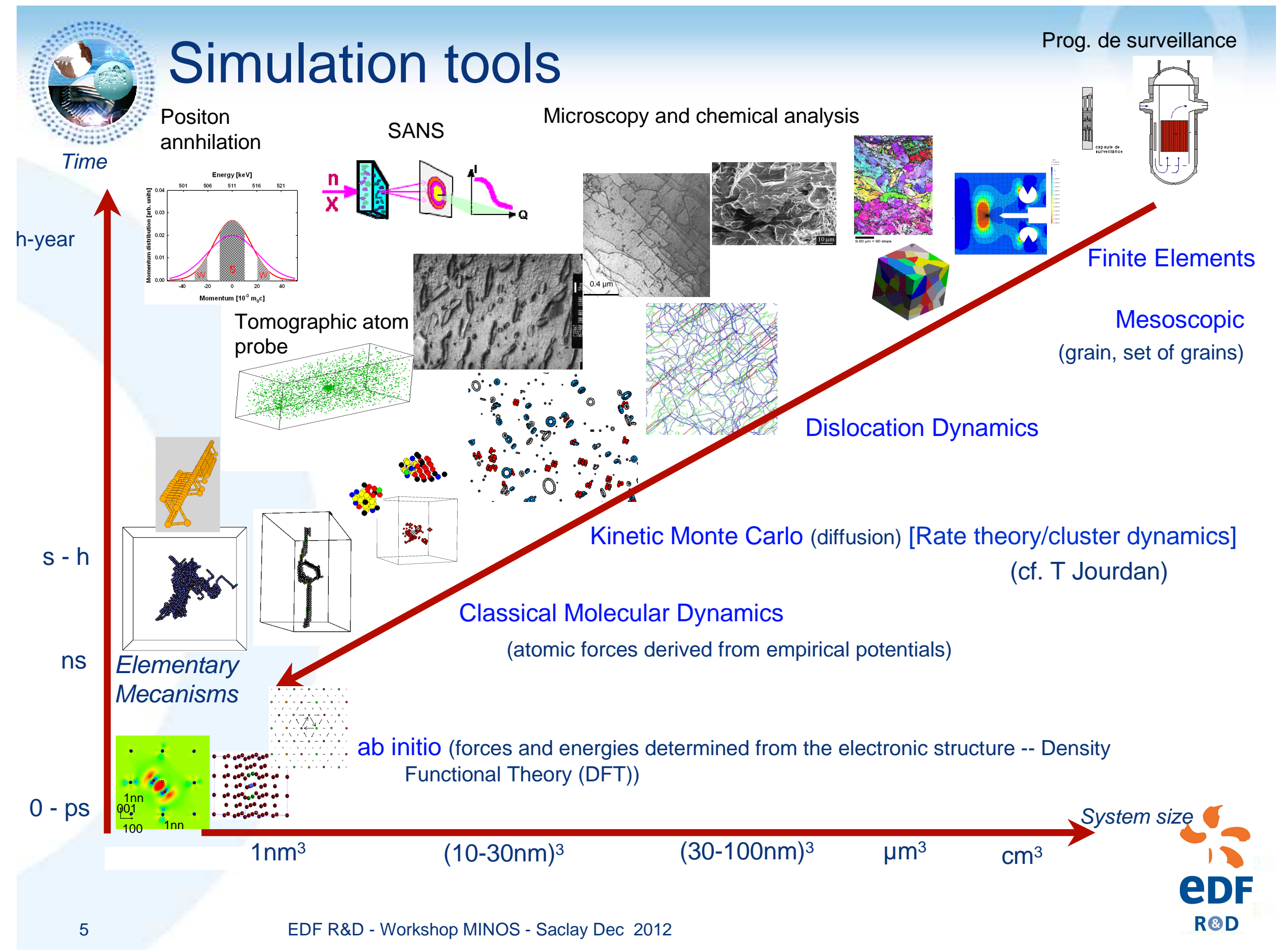




\section{Atomic Kinetic Monte Carlo of microstructure evolution}

\section{Objective:}

Simulation formation of solute rich complexes (observed by TAP) under irradiation

\section{Ab initio}

Solute interactions $(\mathrm{Cu}, \mathrm{Ni}, \mathrm{Mn}, \mathrm{Si})$ (interface energies,

mixing energies ...)

\section{Experimental data and}

Thermodynamical data

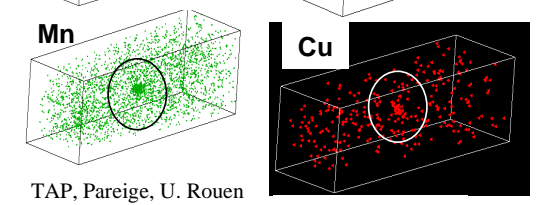

$15 \times 15 \times 50 \mathrm{~nm}$
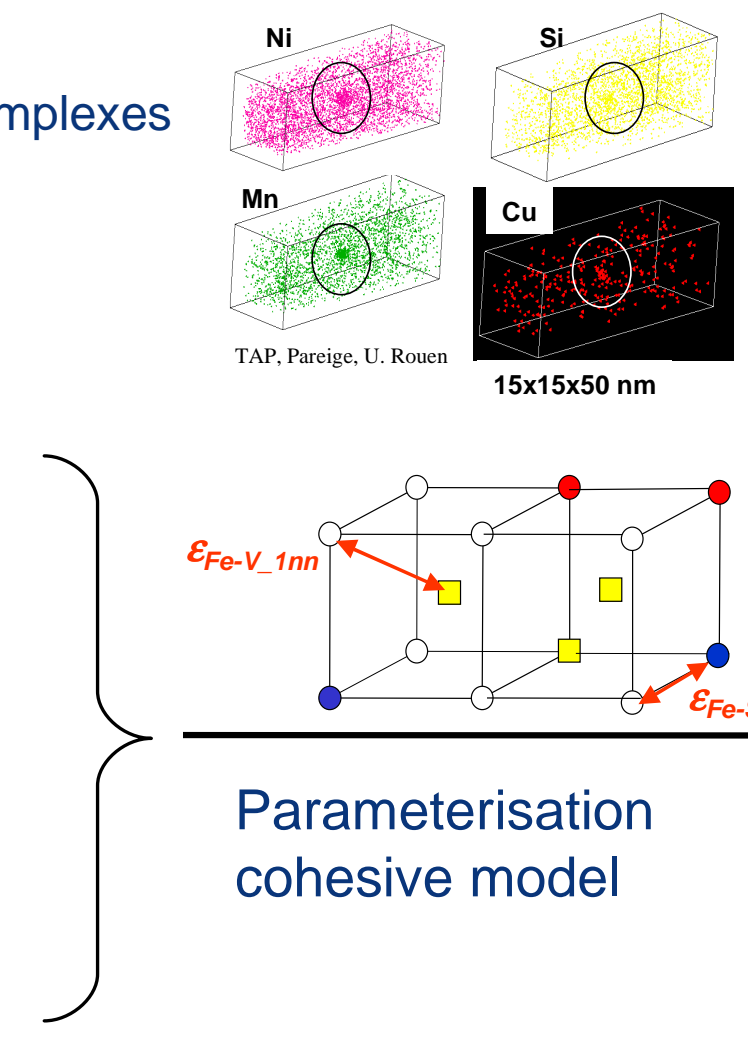

Parameterisation cohesive model
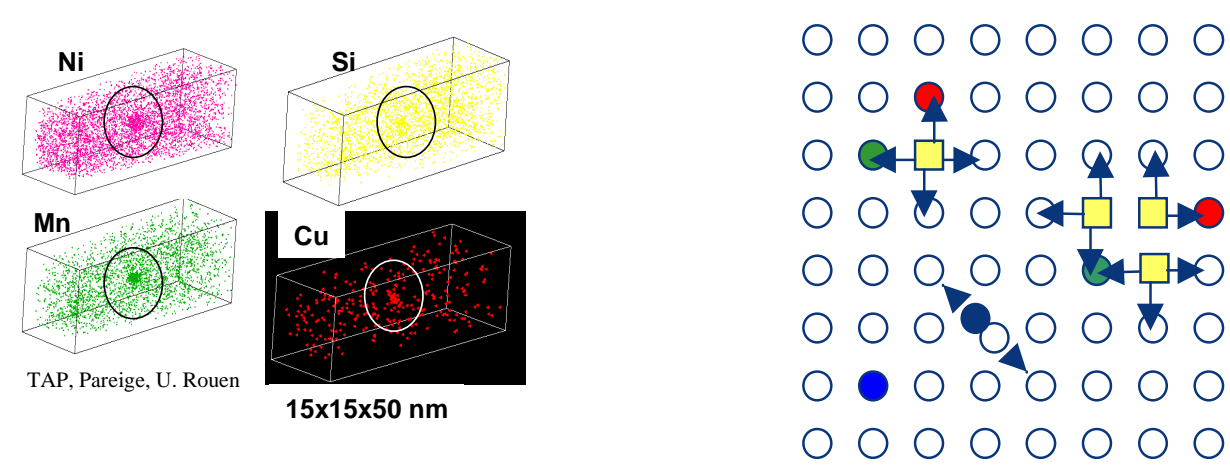

AKMC

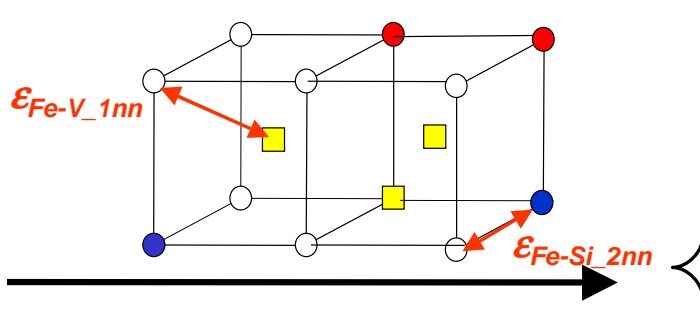

Solute diffusion by

- vacancy mechanisms

- interstitial mechanisms

$$
\begin{gathered}
\left.E_{\text {mixing }}=-4 \varepsilon_{(F e-F e)}^{(1)}-3 \varepsilon_{(F e-F e)}^{(2)}+8 \varepsilon_{(F e-X)}^{(1)}+6 \varepsilon_{(F e-X)}^{(2)}-4 \varepsilon_{(X-X)}^{(1)}-3 \varepsilon^{(2)} / X-X\right) \\
E_{\text {formation }}\left(V^{Z}\right)=8 \varepsilon_{(V-Z)}^{(1)}+6 \varepsilon_{(V-Z)}^{(2)}-4 \varepsilon_{(Z-Z)}^{(1)}-3 \varepsilon_{(Z-Z)}^{(2)} \\
E_{\text {binding }(V-X)}^{(1)}=\varepsilon_{(F e-V)}^{(1)}+\varepsilon_{(F e-X)}^{(1)}-\varepsilon_{(F e-F e)}^{(1)}-\varepsilon_{(V-\not)}^{(1)}
\end{gathered}
$$

\section{Experimental validation: TAP, SANS, SAXS, PA, TEP}




\section{Atomistic Kinetic Monte Carlo (AKMC)}

- Treatment of multi-component systems on a rigid lattice

- Substitutional elements

- Interstitial elements

Code: LAKIMOCA

- Diffusion by 1 nn jumps

- Via vacancies

- Via interstitials

Jump Probability: $\quad \Gamma_{X}=v_{X} \exp \left(-\frac{E a}{k T}\right) \quad v_{X}=$ attempt frequency

Vincent et al. NIMB 255 (2007) 78 Vincent et al. JNM 382 (2008) 154

- Residence Time Algorithm applied to all events

- Vacancy and Interstitial jumps

- Frenkel Pairs and Cascade flux for irradiation

Average time step: $\Delta t=\frac{1}{\sum_{j, k} \Gamma_{j k}}$
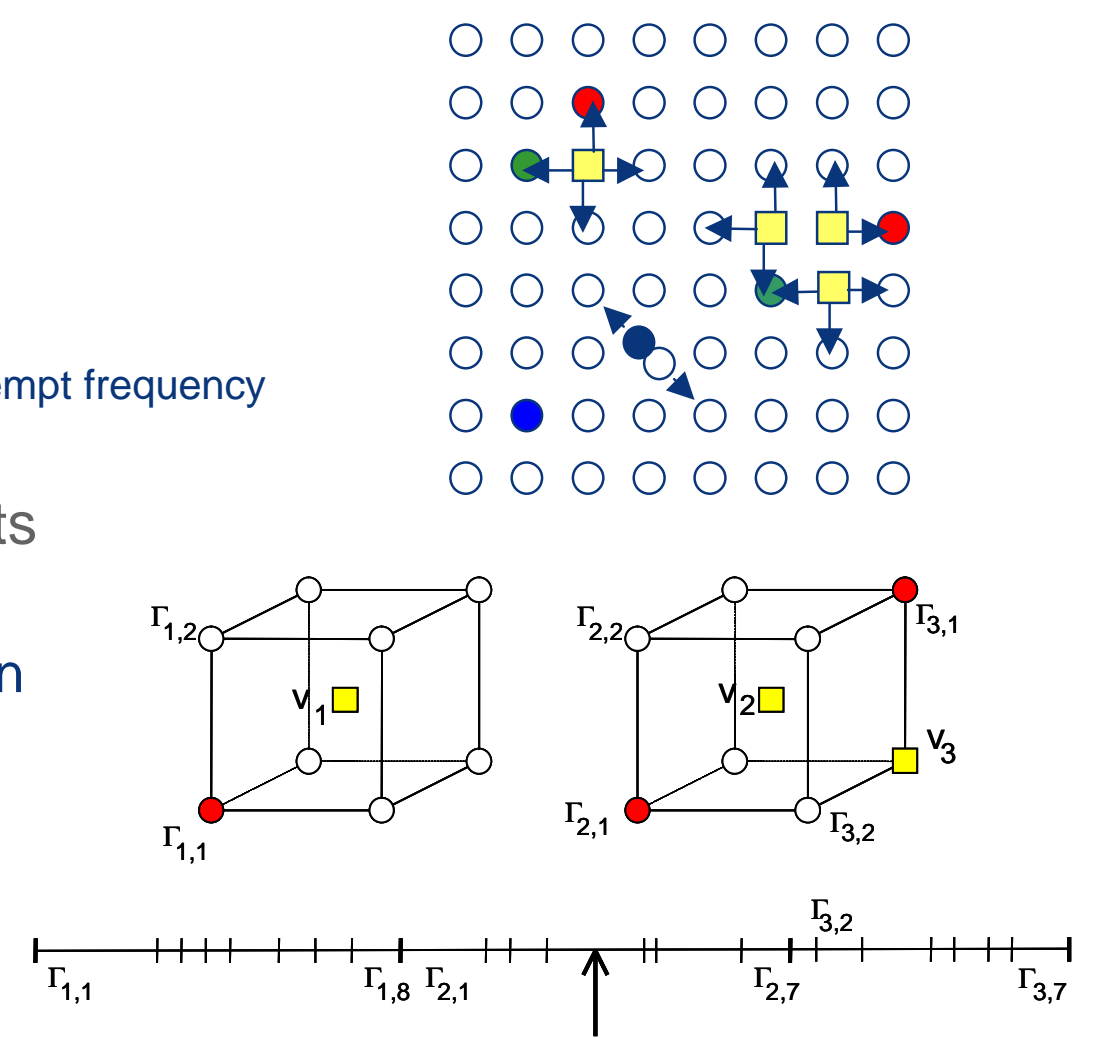

$\rightarrow$ Environment dependant form of activation energy Ea

$$
E a=E a\left(X_{i}\right)+\frac{E f-E i}{2}
$$




\section{AKMC irradiation simulation conditions}

For electron irradiation: Frenkel Pair (FP) flux

For neutron irradiation: flux of

- $20 \mathrm{keV}$ and $100 \mathrm{keV}$ cascades debris obtained by Molecular Dynamics

(R. Stoller, J. Nucl. Mater. 307-311 (2002) 935)

- Frenkel Pairs

Typical simulation box:

$1.01 \times 10^{-17} \mathrm{~cm}^{3}$ boxes

$8.6510^{6}$ atoms

surface

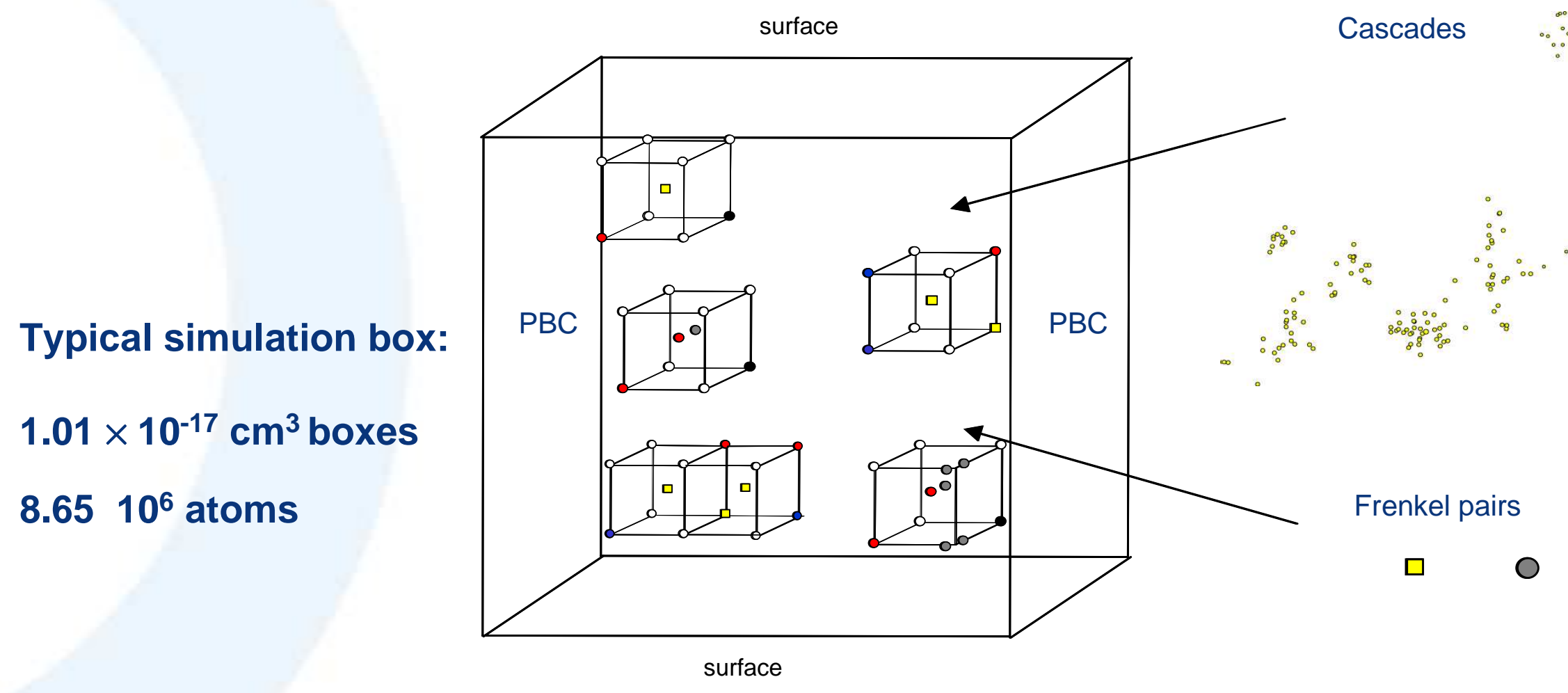

Cascades 


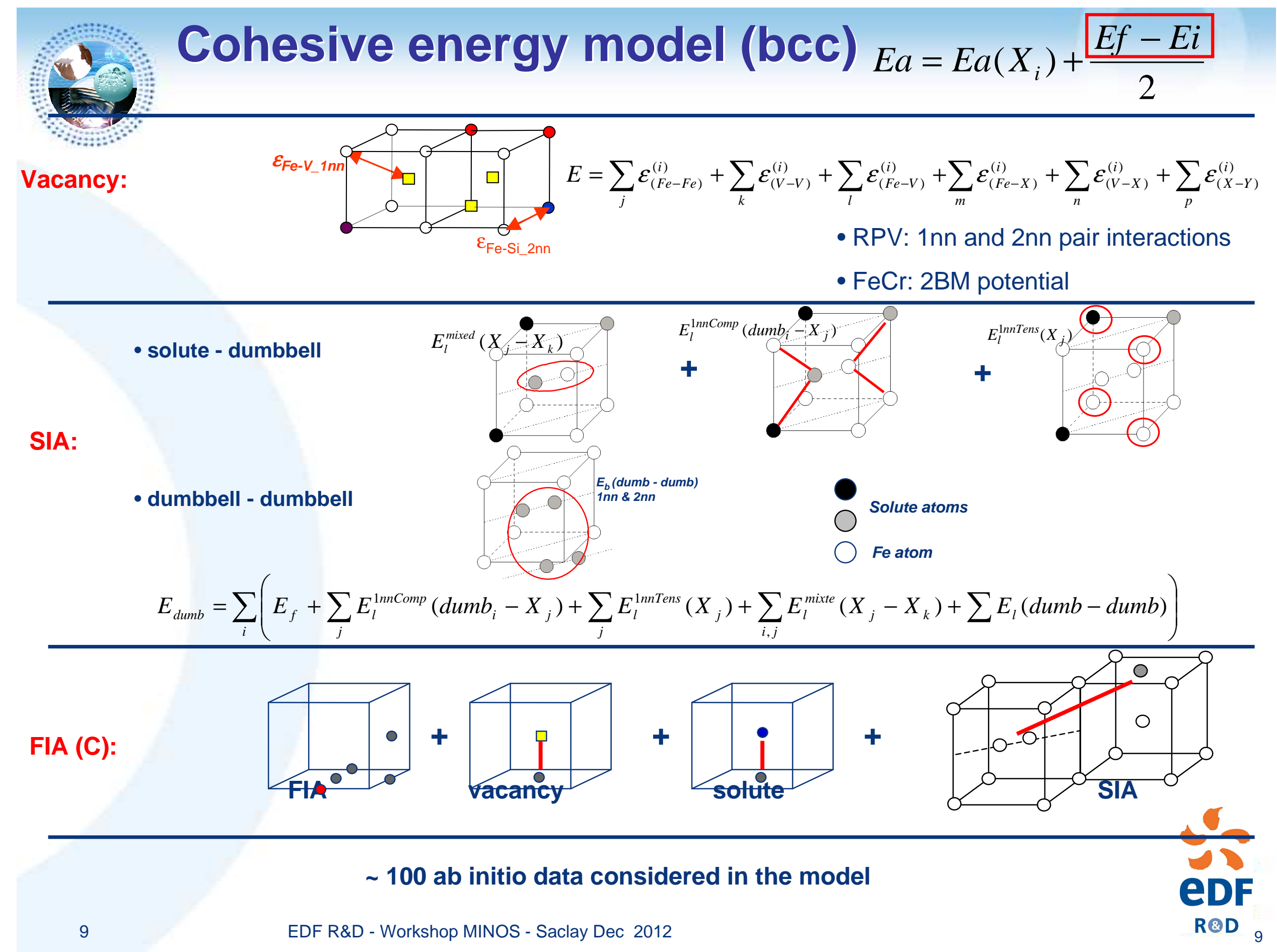




\section{Cohesive model: $\varepsilon_{\mathrm{X}-\mathrm{Y}}$ and $\varepsilon_{\mathrm{V}-\mathrm{X}}$ determination}

$$
\begin{aligned}
& \text { Binary alloys } \\
& \text { - } \\
& E_{\text {mélange }}=-4 \varepsilon_{(F e-F e)}^{(1)}-3 \varepsilon_{(F e-F e)}^{(2)}+8 \varepsilon_{(F e-X)}^{(1)}+6 \varepsilon_{(F e-X)}^{(2)}-4 \varepsilon_{(X-X)}^{(1)}-3 \varepsilon_{(X-X)}^{(2)} \\
& \text { • } \\
& E_{\text {int } \operatorname{erface}(100)}=-2 \varepsilon_{(F e-F e)}^{(1)}-\varepsilon_{(F e-F e)}^{(2)}+4 \varepsilon_{(F e-X)}^{(1)}+2 \varepsilon_{(F e-X)}^{(2)}-2 \varepsilon_{(X-X)}^{(1)}-\varepsilon_{(X-X)}^{(2)} \\
& E_{\text {cohésion }}(Z)=4 \varepsilon_{(Z-Z)}^{(1)}+3 \varepsilon_{(Z-Z)}^{(2)} \\
& \text { - } E_{\text {formation }}\left(\text { lac }{ }^{Z}\right)=8 \varepsilon_{(l a c-Z)}^{(1)}+6 \varepsilon_{(l a c-Z)}^{(2)}-4 \varepsilon_{(Z-Z)}^{(1)}-3 \varepsilon_{(Z-Z)}^{(2)} \\
& \text { - } E_{\text {liaison }(l a c-l a c)}^{(i)}=2 \varepsilon_{(F e-l a c)}^{(i)}-\varepsilon_{(F e-F e)}^{(i)}-\varepsilon_{(l a c-l a c)}^{(i)} \\
& E_{\text {liaison }(l a c-X)}^{(1)}=\varepsilon_{(F e-l a c)}^{(1)}+\varepsilon_{(F e-X)}^{(1)}-\varepsilon_{(F e-F e)}^{(1)}-\varepsilon_{(l a c-X)}^{(1)} \\
& \text { Ternary alloys... } \\
& E_{\text {liaison }(X-Y)}^{(i)}=\varepsilon_{(F e-X)}^{(i)}+\varepsilon_{(F e-Y)}^{(i)}-\varepsilon_{(F e-F e)}^{(i)}-\varepsilon_{(X-Y)}^{(i)} \\
& i=1 \text { or } 2 \\
& \mathrm{X}, \mathrm{Y}=\text { solute atoms }
\end{aligned}
$$




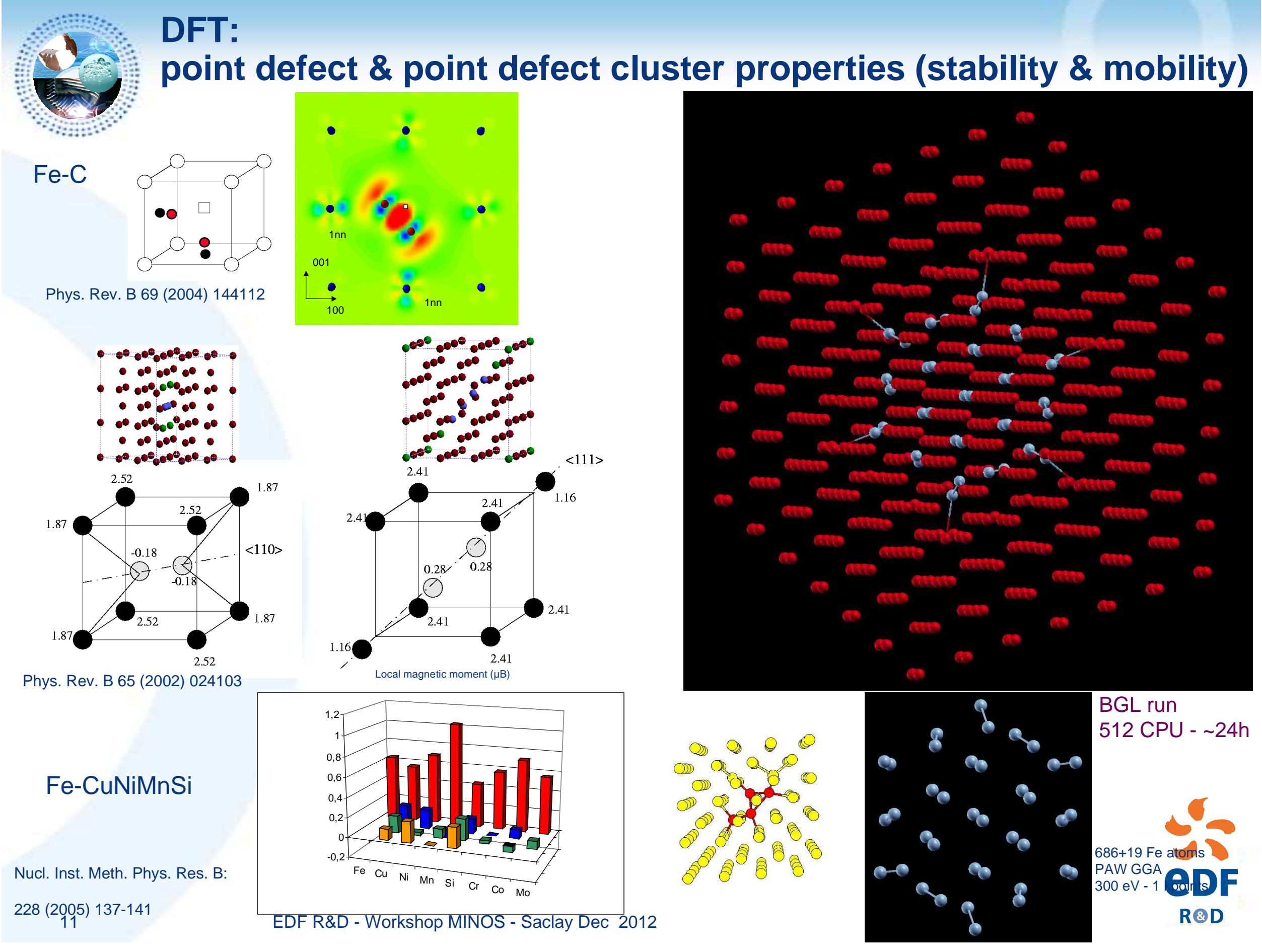




\section{Neutron irradiation of FeCuNiMnSi alloys Medium term evolution by atomic Kinetic Monte Carlo}

Fe-0.2Cu-0.53Ni-1.26Mn-0.63Si (at.\%) at $300^{\circ} \mathrm{C}$

Flux: $6.510^{-5}$ dpa.s $^{-1}$

Dose: $1.310^{-3} \mathrm{dpa}$

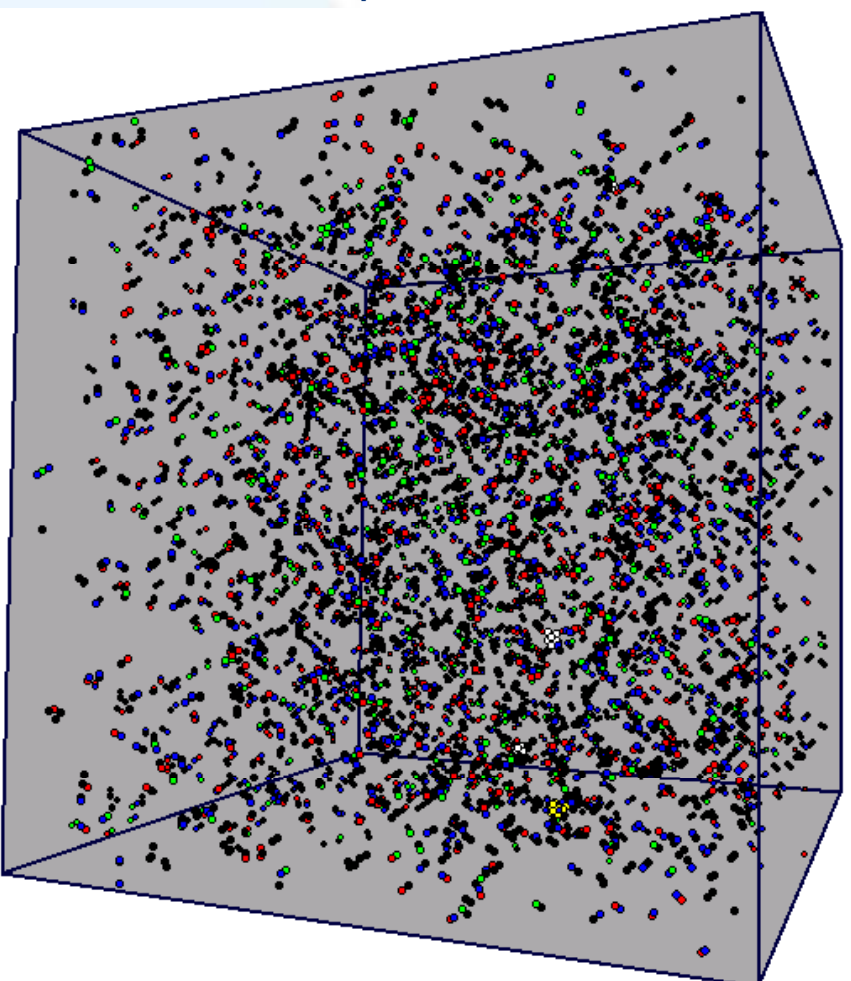

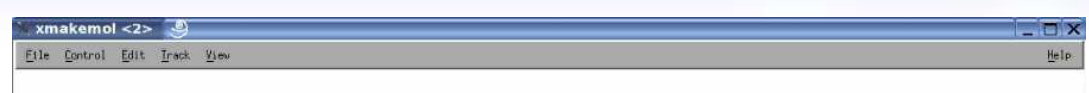

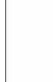

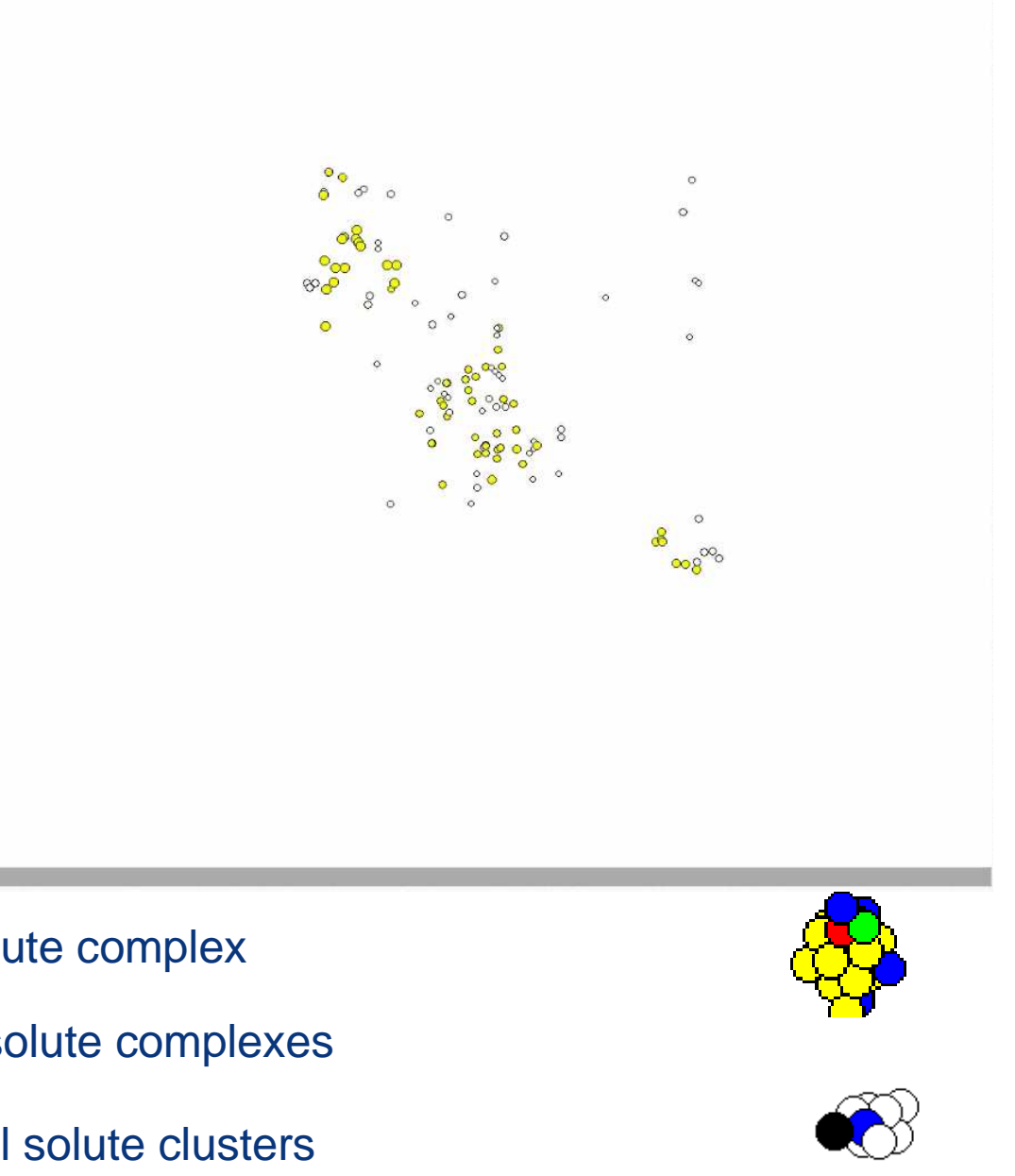

- $\mathrm{Cu} \bigcirc \mathrm{Ni}$

- Si $\bigcirc \mathrm{V}$

- Mn $\bigcirc$ SIA
V-solute complex

SIA-solute complexes

Small solute clusters
Point defect clusters = germs for precipitation

[>1 month on $1 \mathrm{CPU}]$
eDF

R\&D 


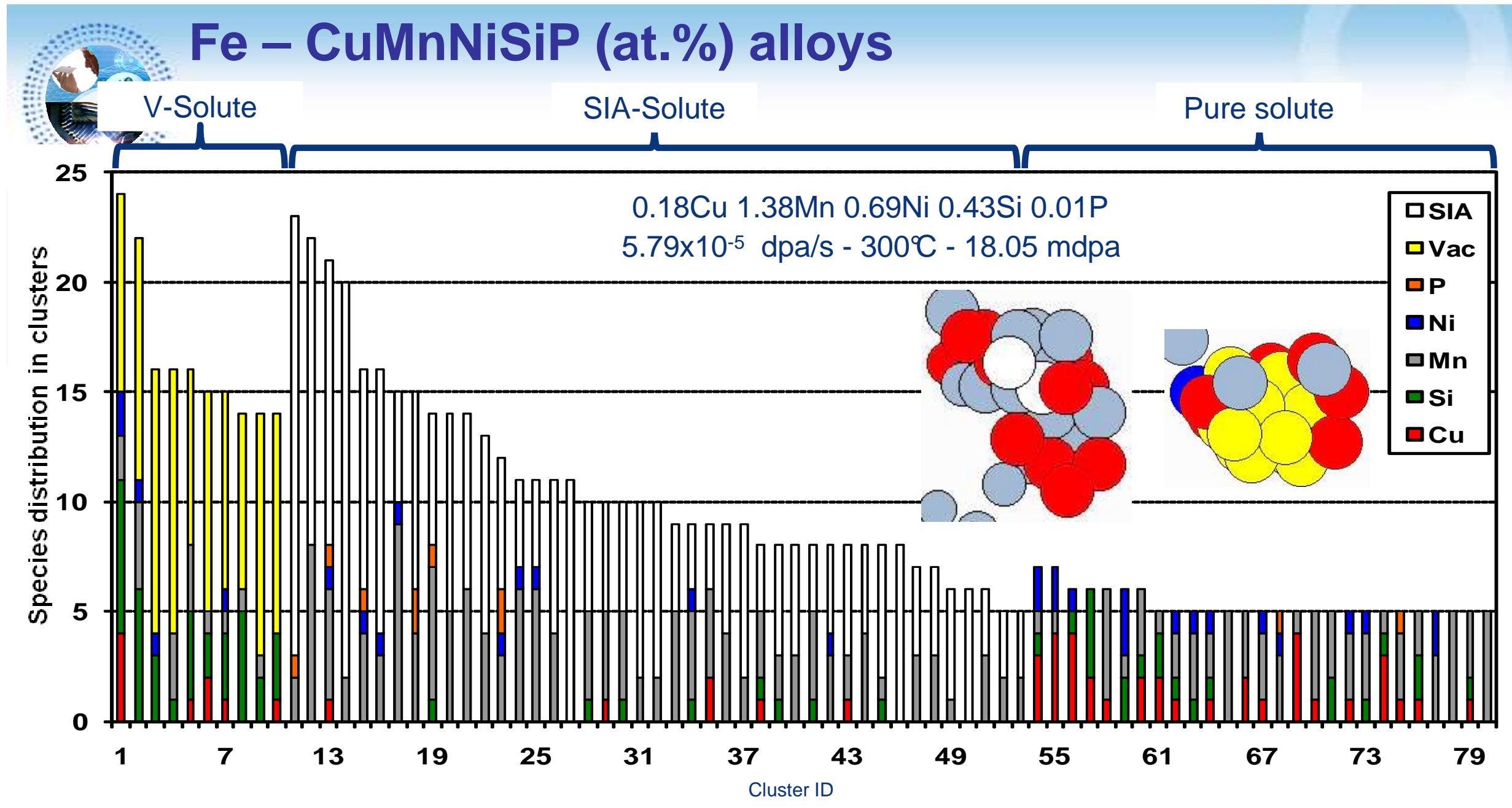

- The biggest solute clusters are associated with PD clusters

- In agreement with induced segregation mechanism to account for solute clusters formation

- Clusters associated with interstitial clusters are enriched in $\mathrm{Mn}$, and $\mathrm{P} / \mathrm{Ni}$

- Clusters associated with vacancy clusters are enriched in Si/Cu/Mn (mostly) and $\mathrm{Ni}$

- I-Solute complexes > V-Solute complexes 


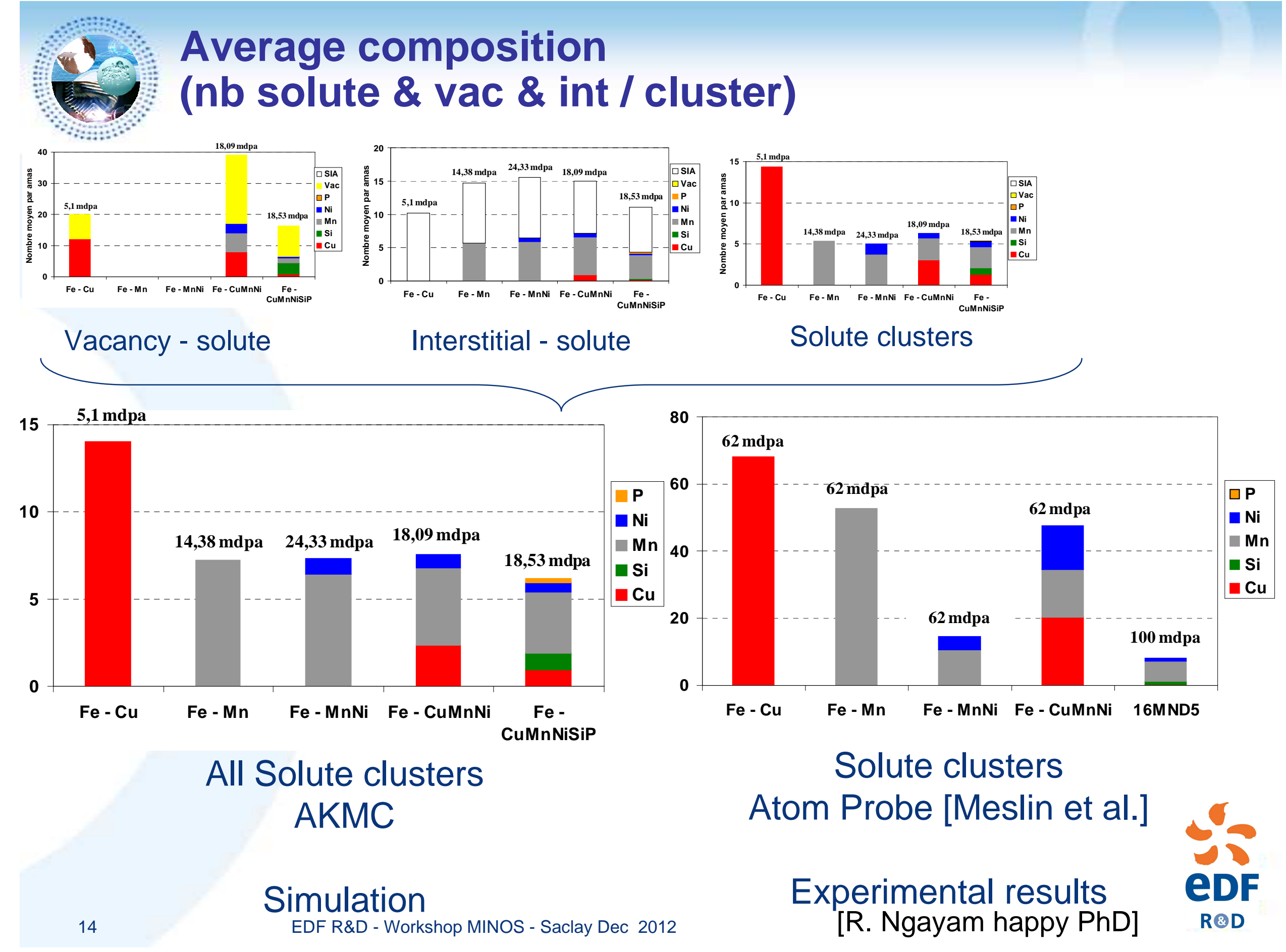




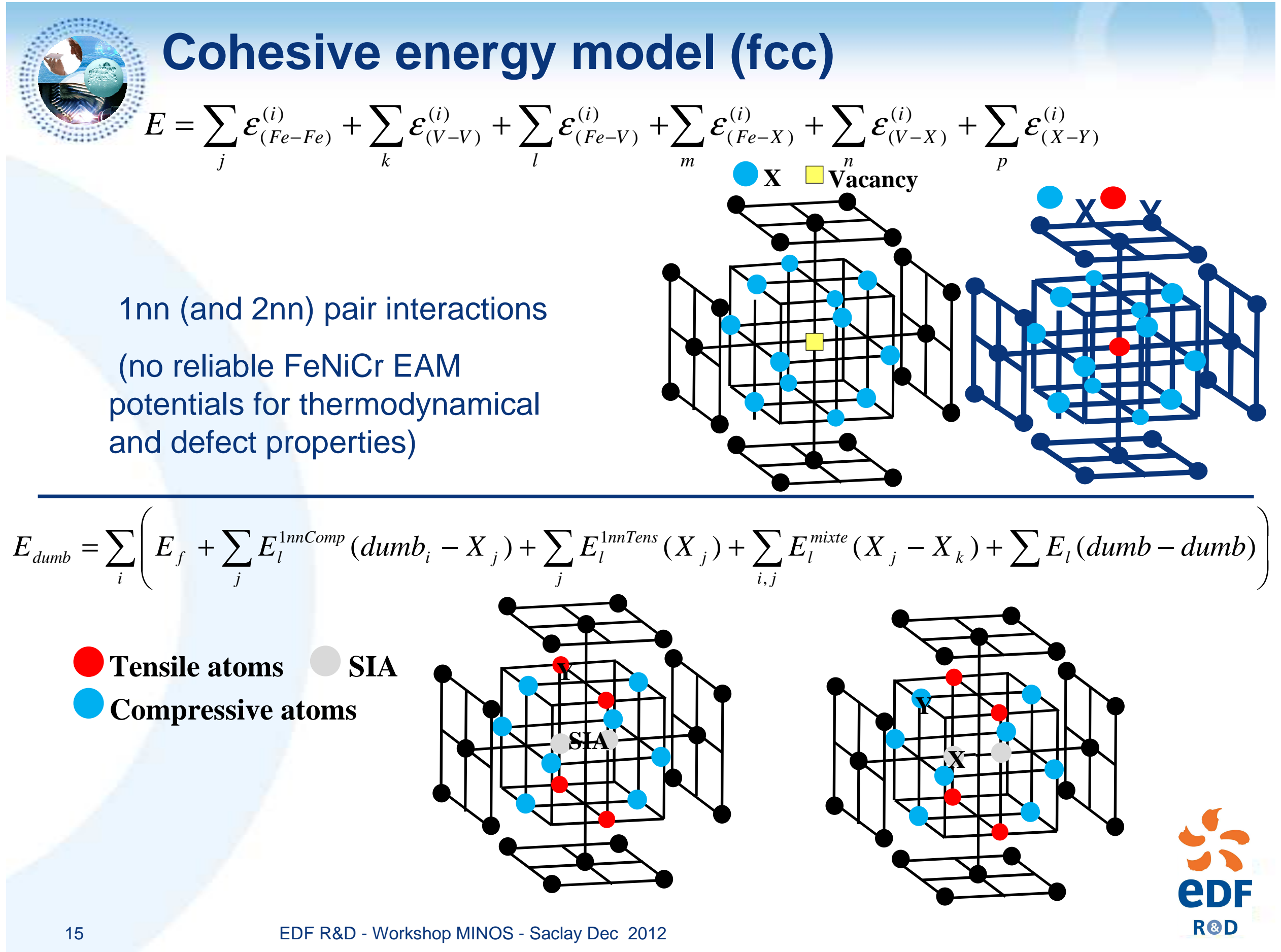




\section{FeNiCr interaction parameters adjustment on DFT data $\left(\mathrm{Fe}_{70} \mathrm{Ni}_{10} \mathrm{Cr}_{20}\right)$}

Chemical interactions in the Bulk

Cohesive energies $\rightarrow \mathrm{X}-\mathrm{X}$ terms

$$
\begin{aligned}
& E_{c}^{F e}=6 \varepsilon_{F e F e} \\
& E_{c}^{N i}=6 \varepsilon_{N i N i} \\
& E_{c}^{C r}=6 \varepsilon_{C r C r}
\end{aligned}
$$

Binding energies in dilute $\mathrm{Y}$-Fe $\rightarrow \mathrm{X}$-Y terms

$$
\begin{gathered}
E_{b}^{\mathrm{NiNi} / \mathrm{Fe}}=-E_{\mathrm{mix}}^{\mathrm{FeNi}} / 12=2 \varepsilon_{\mathrm{FeNi}}-\varepsilon_{\mathrm{FeFe}}-\varepsilon_{\mathrm{NiNi}} \\
E_{b}^{\mathrm{CrCr} / \mathrm{Fe}}=2 \varepsilon_{\mathrm{FeCr}}-\varepsilon_{\mathrm{FeFe}}-\varepsilon_{\mathrm{CrCr}} \\
E_{b}^{\mathrm{NiCr} / \mathrm{Fe}}=\varepsilon_{\mathrm{FeCr}}+\varepsilon_{\mathrm{FeN} i}-\varepsilon_{\mathrm{NiCr}}-\varepsilon_{\mathrm{FeFe}}
\end{gathered}
$$

\section{Vacancv-solute interactions}
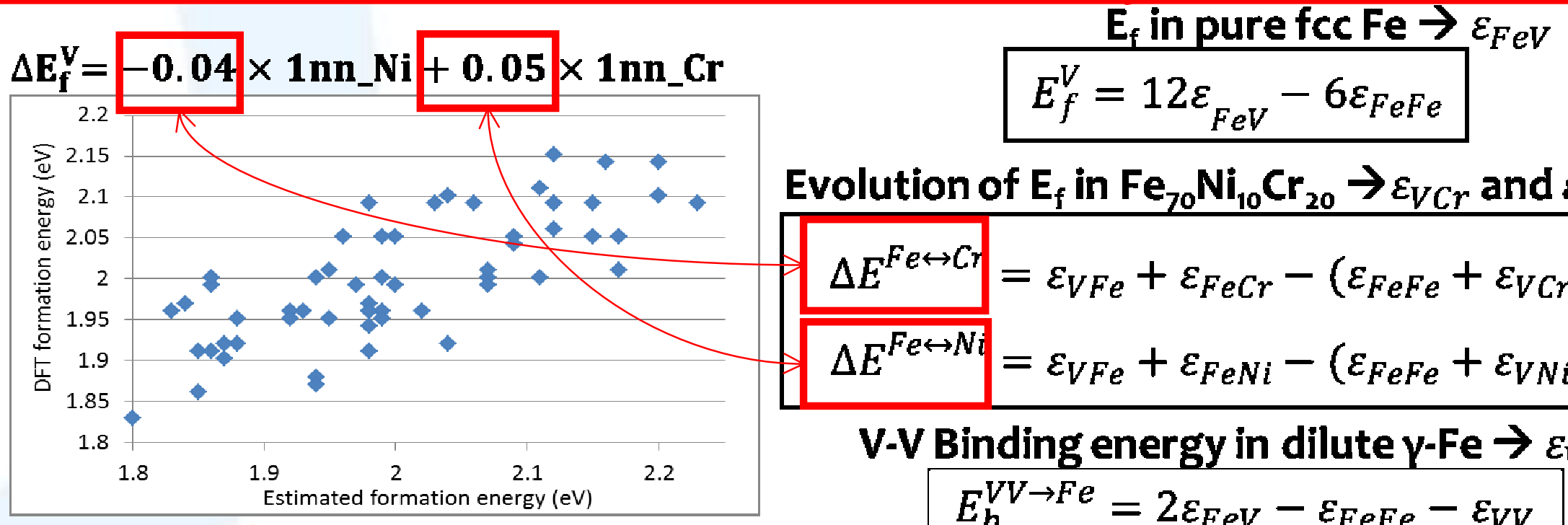

Evolution of $\mathrm{E}_{\mathrm{f}}$ in $\mathrm{Fe}_{70} \mathrm{Ni}_{10} \mathrm{Cr}_{20} \rightarrow \varepsilon_{V C r}$ and $\varepsilon_{V N i}$ $\Delta E^{F e \leftrightarrow C r}=\varepsilon_{V F e}+\varepsilon_{F e C r}-\left(\varepsilon_{F e F e}+\varepsilon_{V C r}\right)$ $\Delta E^{F e \leftrightarrow N i}=\varepsilon_{V F e}+\varepsilon_{F e N i}-\left(\varepsilon_{F e F e}+\varepsilon_{V N i}\right)$

$\mathrm{V}$-V Binding energy in dilute $\mathrm{\gamma}$-Fe $\rightarrow \varepsilon_{V V}$ $E_{b}^{V V \rightarrow F e}=2 \varepsilon_{F e V}-\varepsilon_{F e F e}-\varepsilon_{V V}$ 


\section{TNES \& RIS profil}

\section{Electron irradiation}

$0.6 \mathrm{dpa}$

TNES

RIS

$723 \mathrm{~K}$

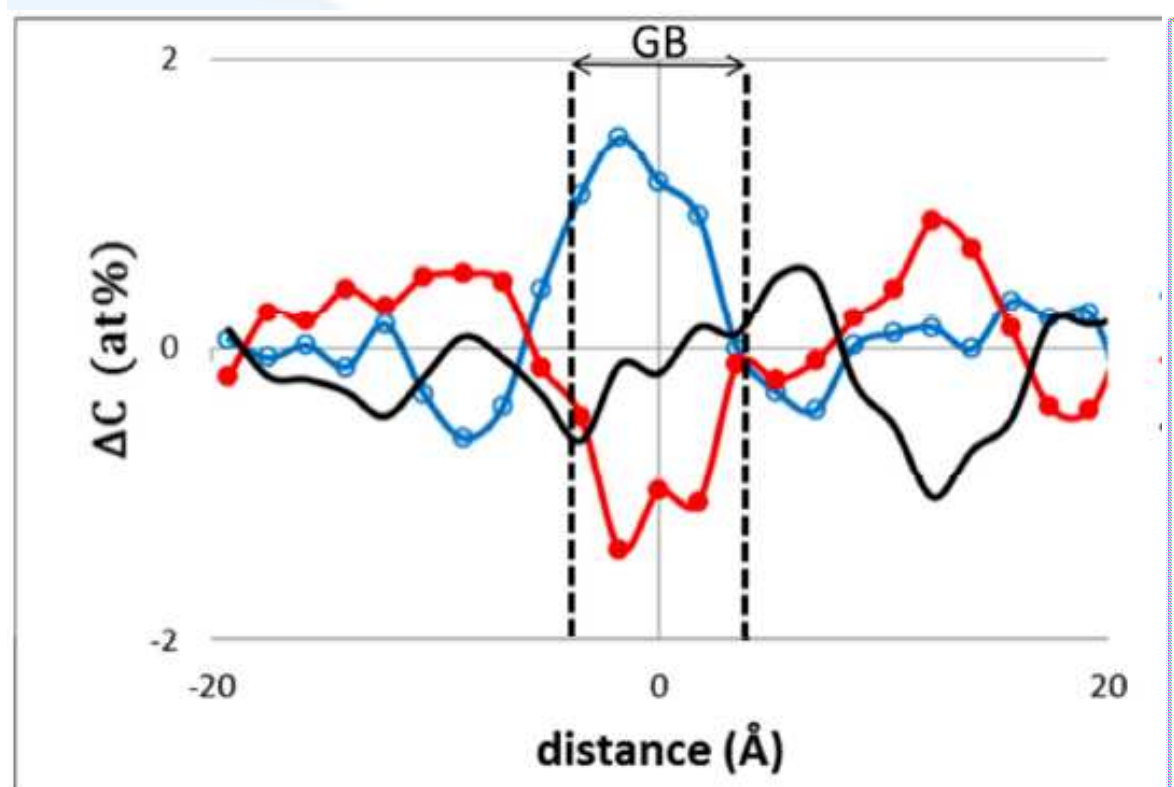

TNES results:

Cr enrichment

Ni depletion

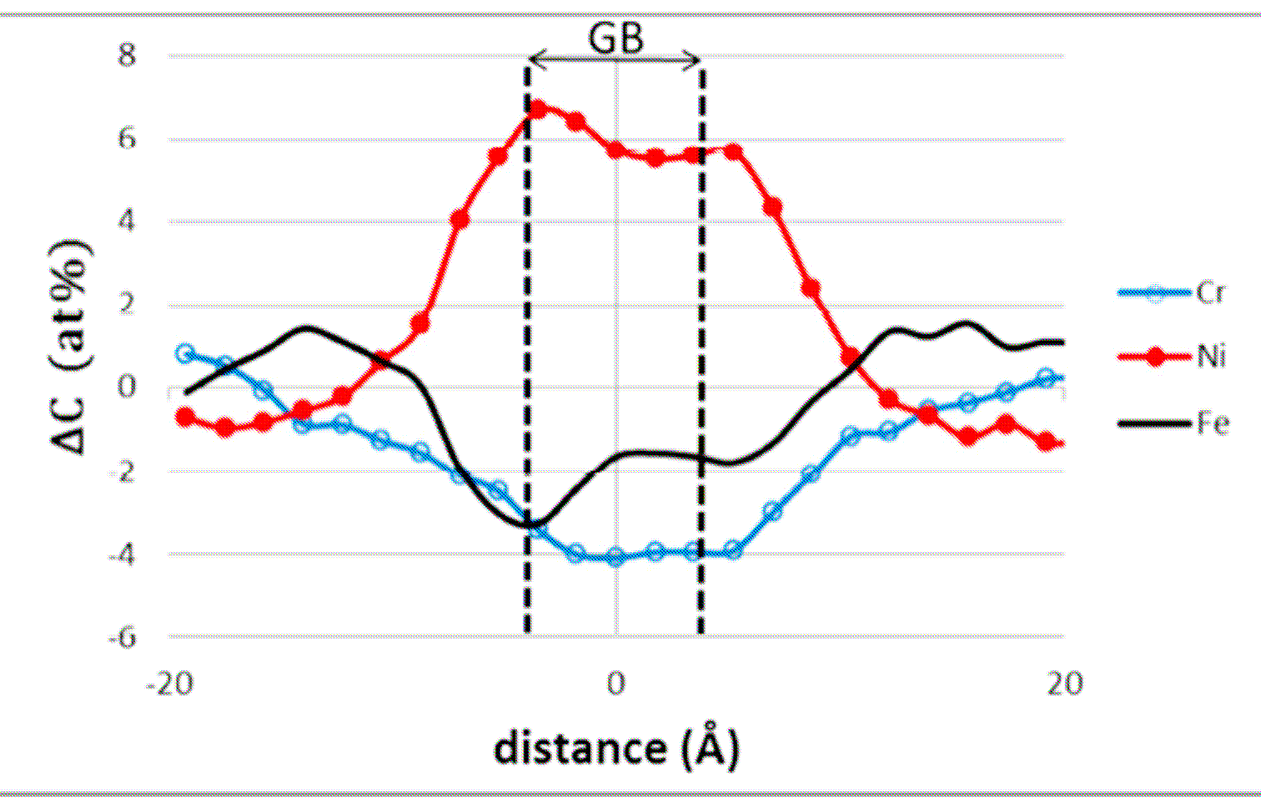

RIS results:

Cr depletion

Ni enrichment

$\rightarrow$ Coherent with experimental results 


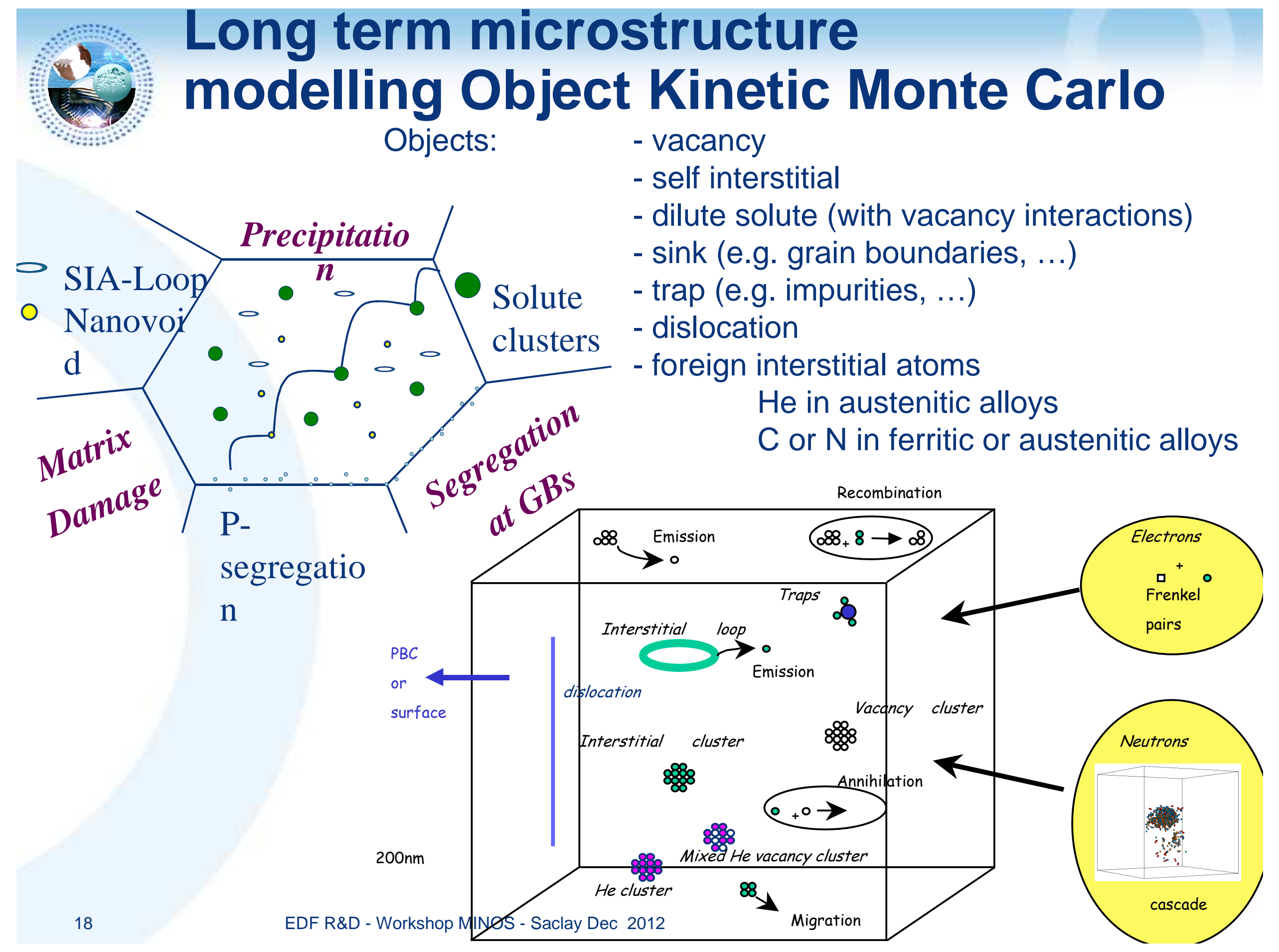



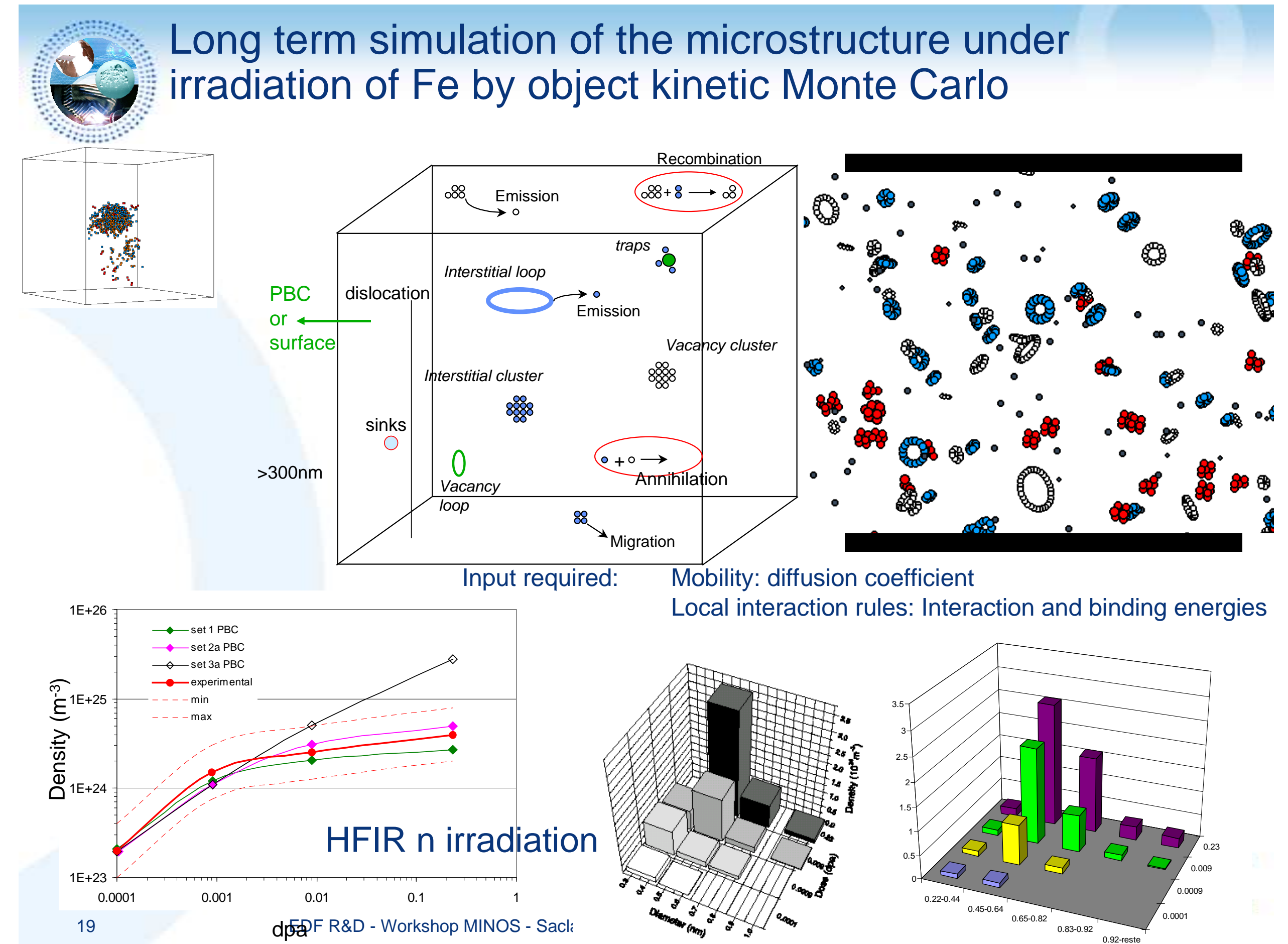

Mobility: diffusion coefficient

Local interaction rules: Interaction and binding energies

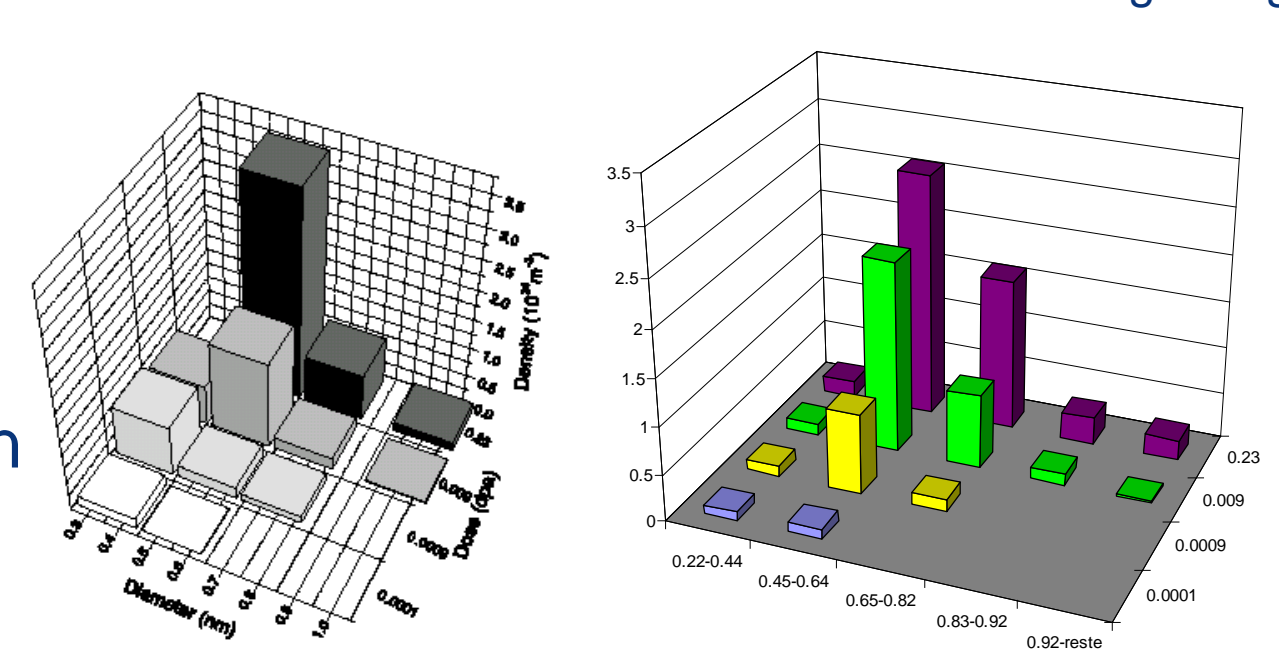




\section{Long term simulation of the microstructure:
Application example: flux effect study in bcc Fe}

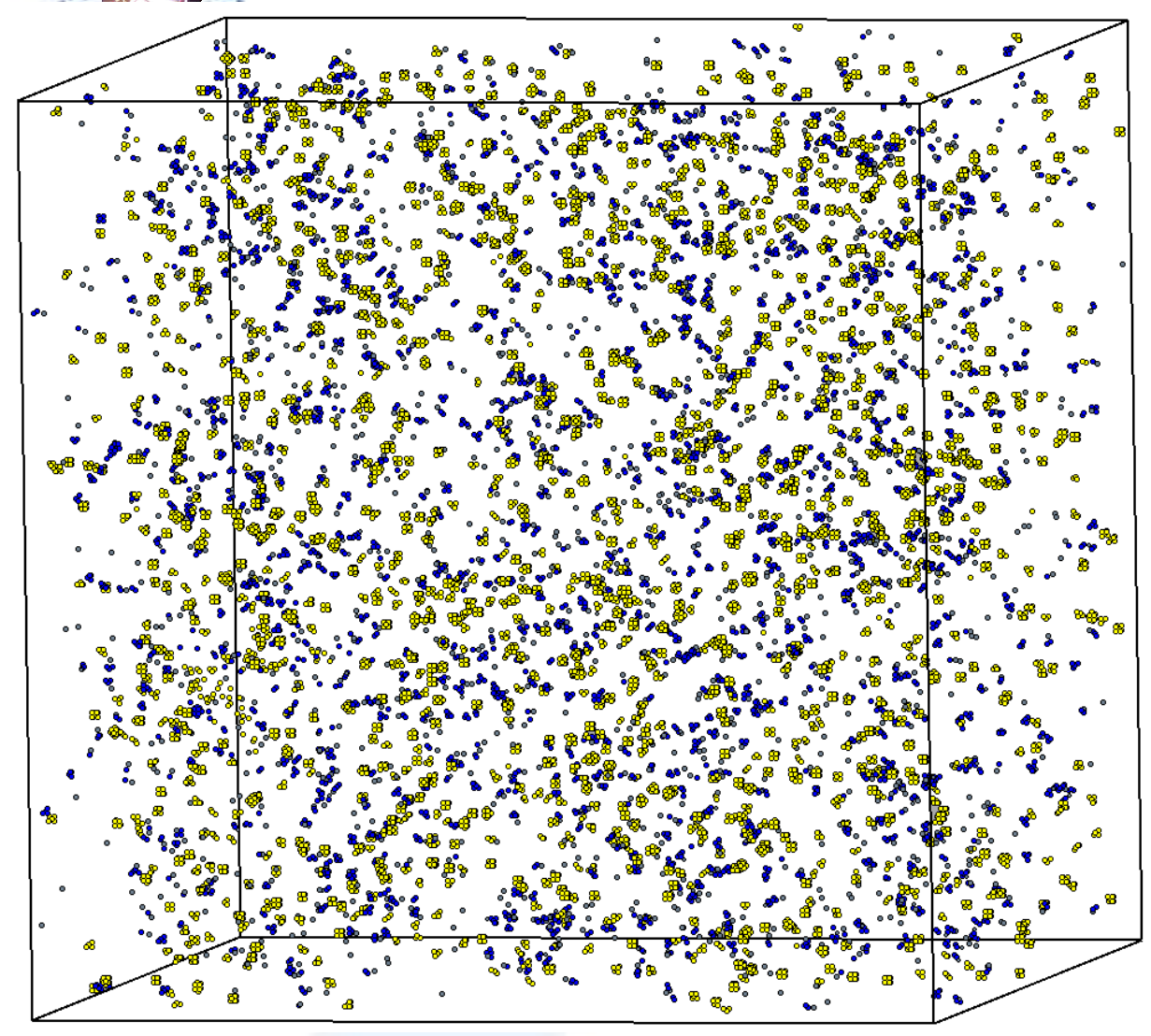

DEFECT POPULATION at $0.1 \mathrm{dpa}$

$\longleftarrow \quad 710^{-5} \mathrm{dpa} / \mathrm{s}$

\section{$710^{-11} \mathrm{dpa} / \mathrm{s}$}

(param Set II)

$343 K$

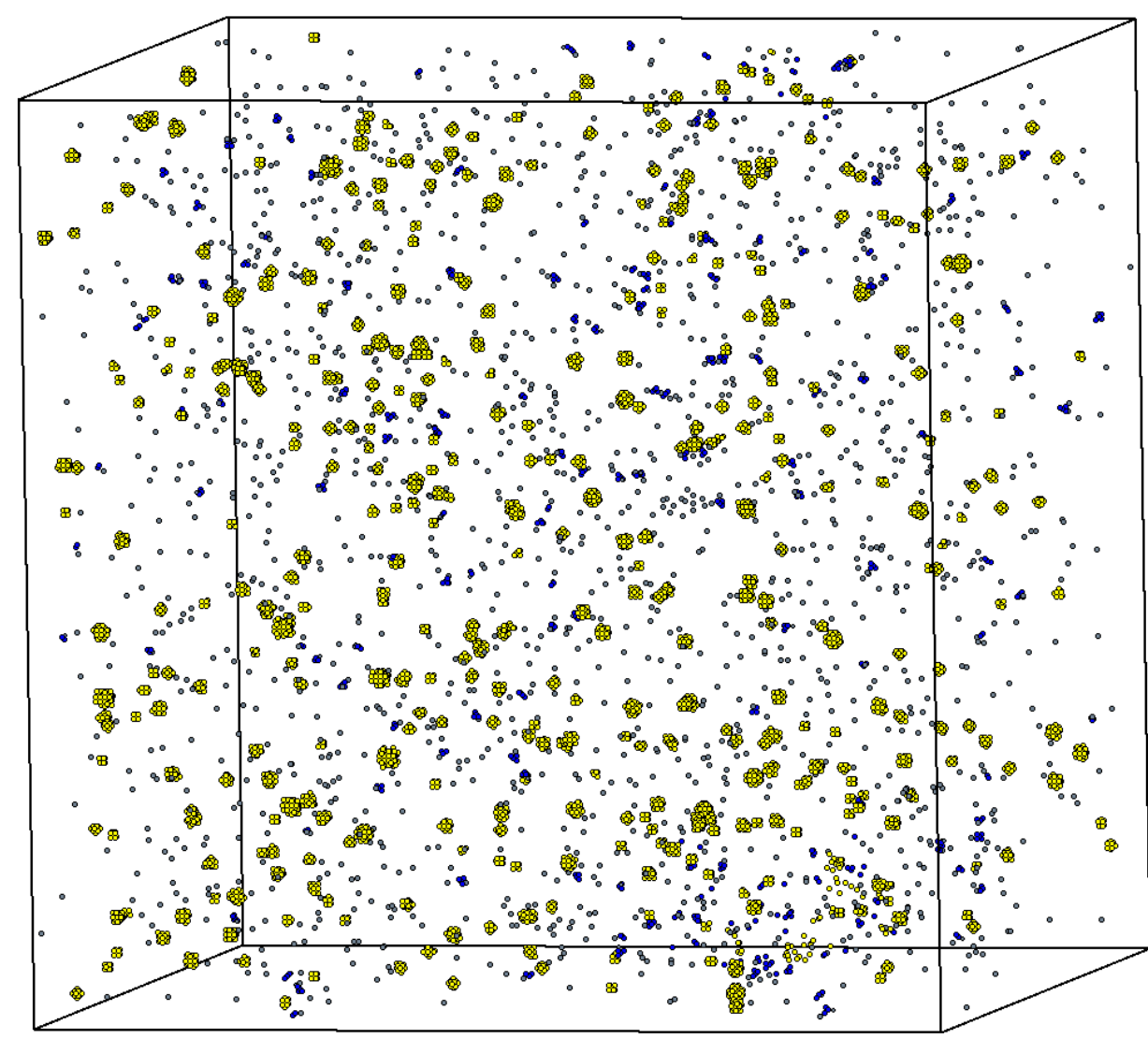




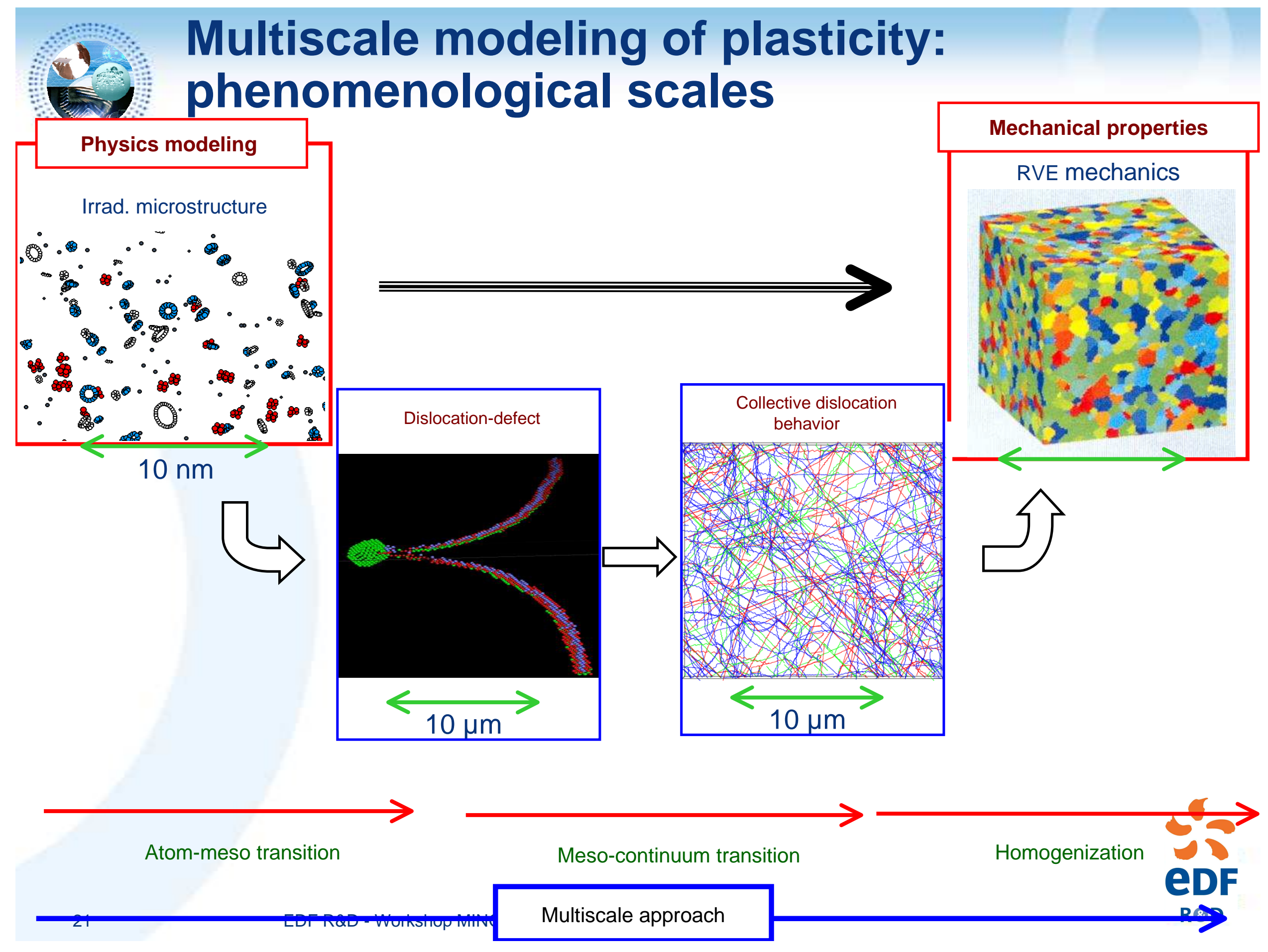




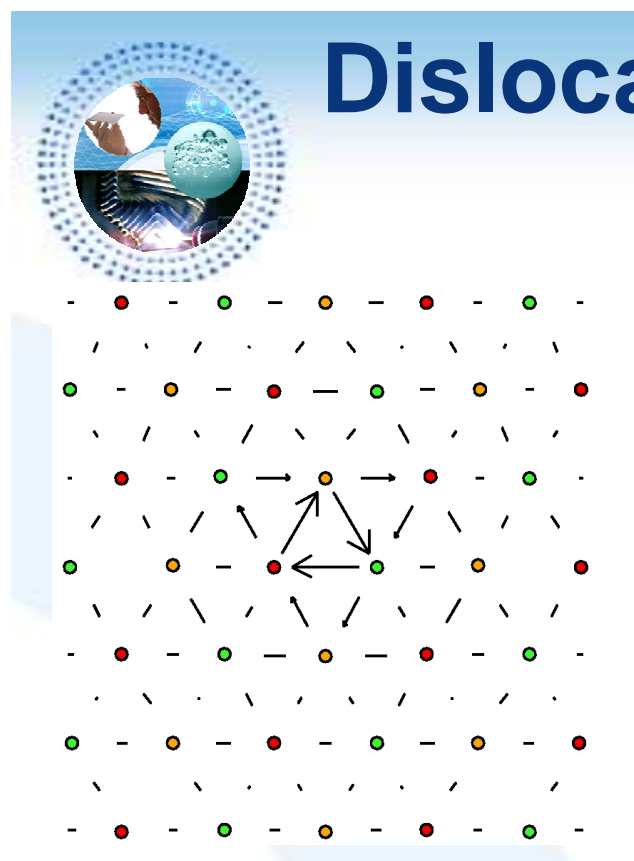

Screw core structure: compact ab initio

EAM: Mendelev03

EAM: Ackland04

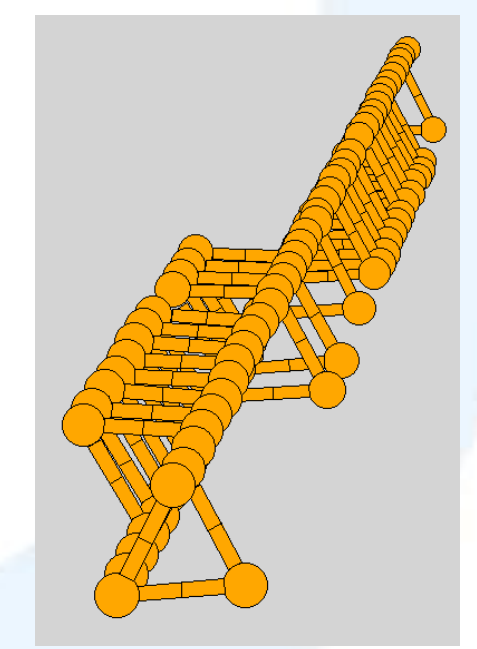

Screw2 2 motion by DK

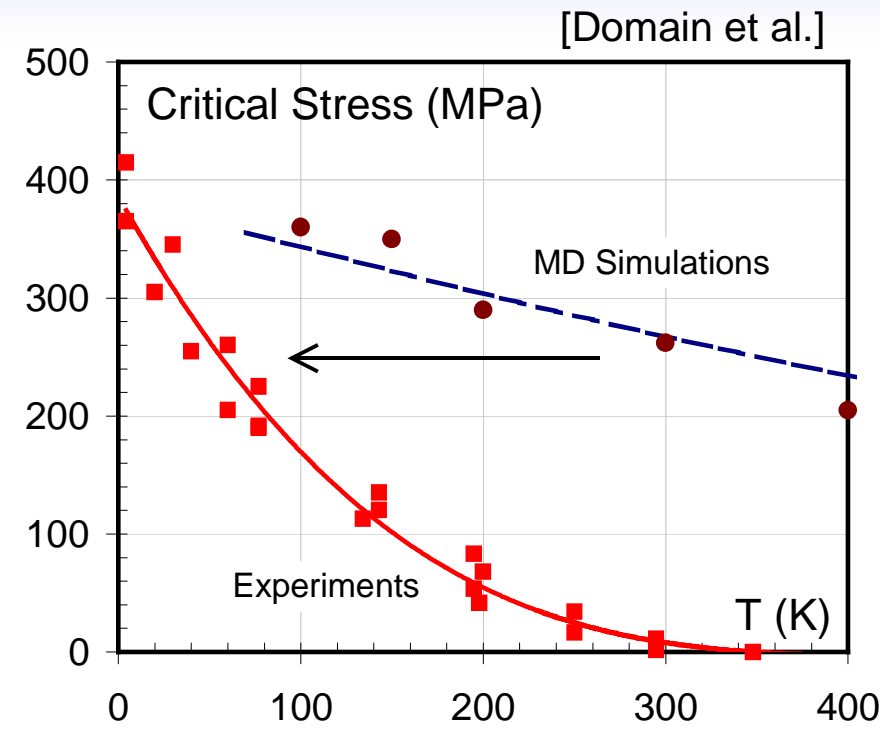

Critical stress for (110) screw dislocation (temperature, strain rate)

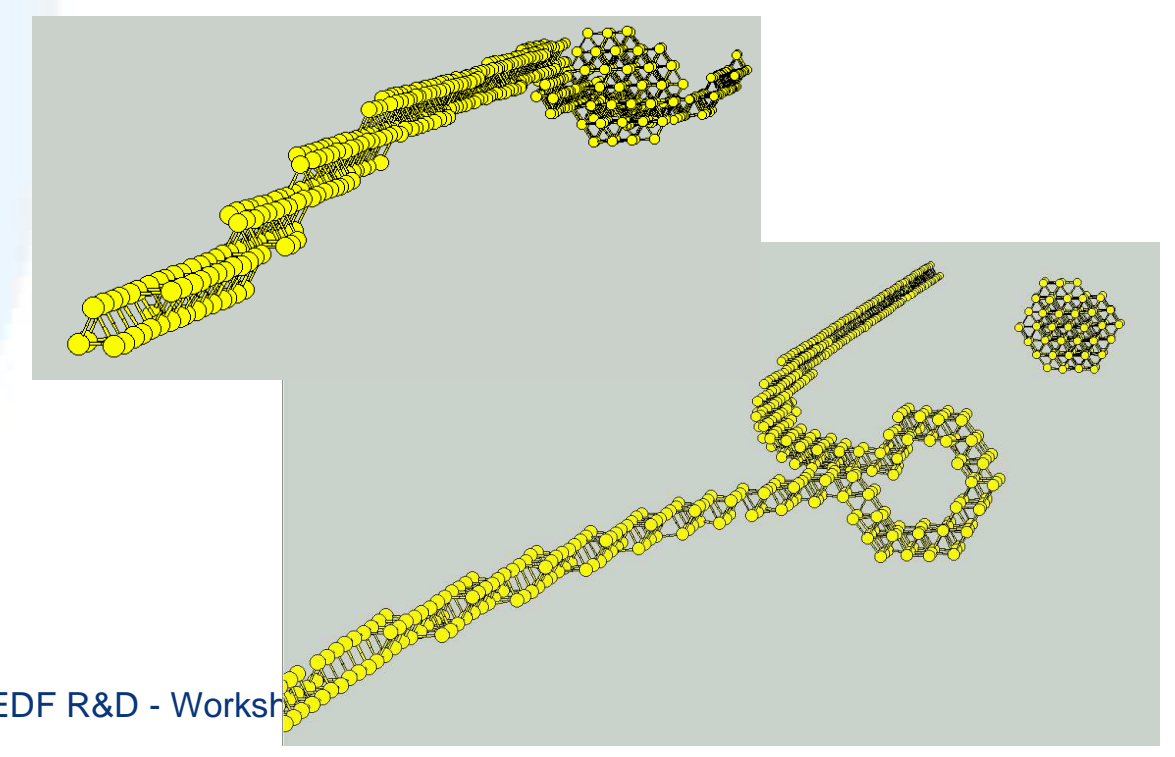

[Terentyev et al.]

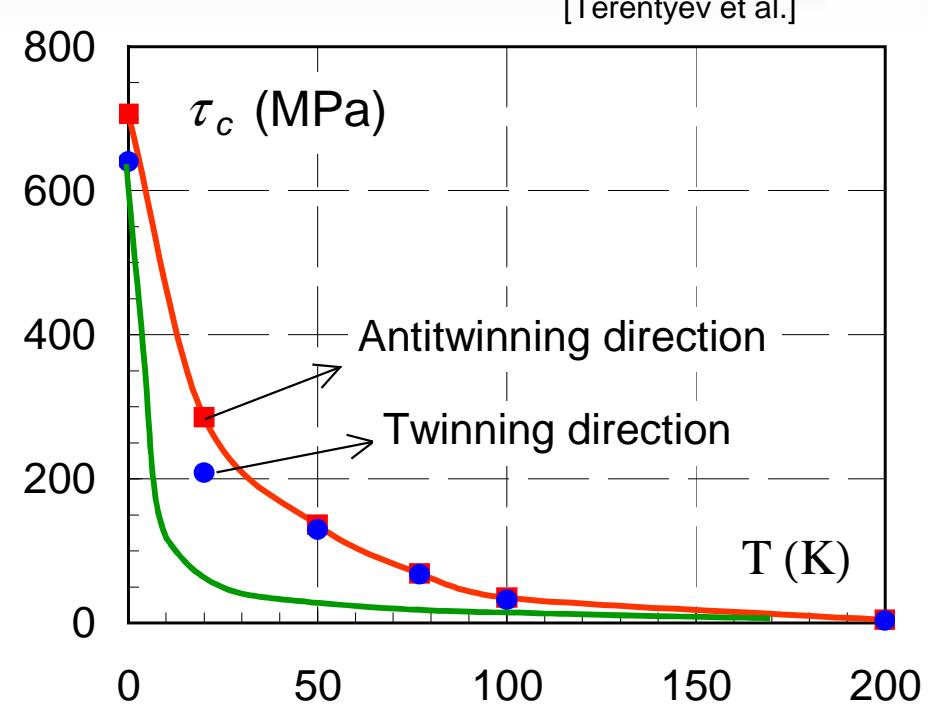

Critical stress for (112) edge dislocation (temperature, strain rate )

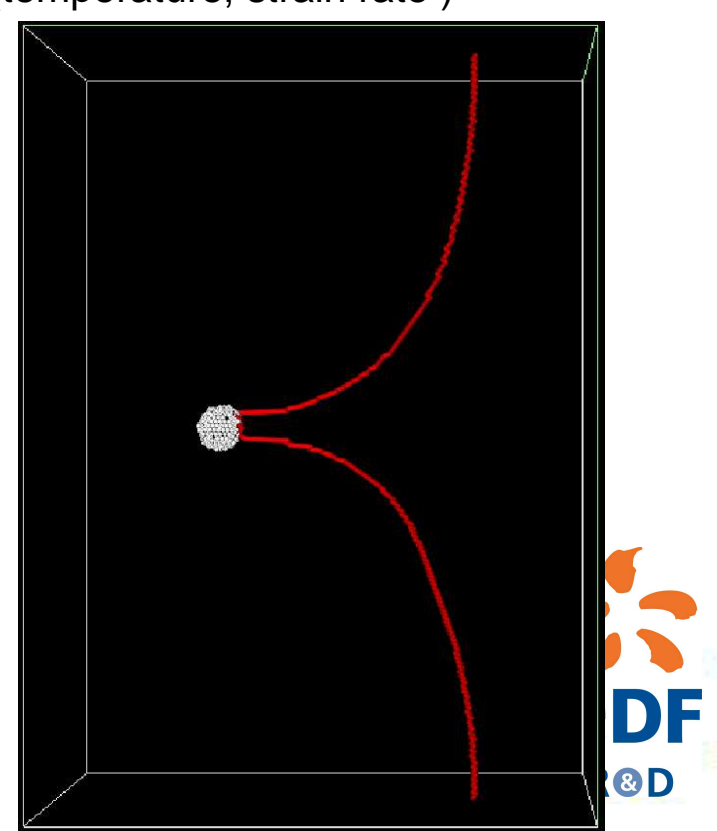




\section{Irradiation strengthening in RPV}

Shear stress (MPa)

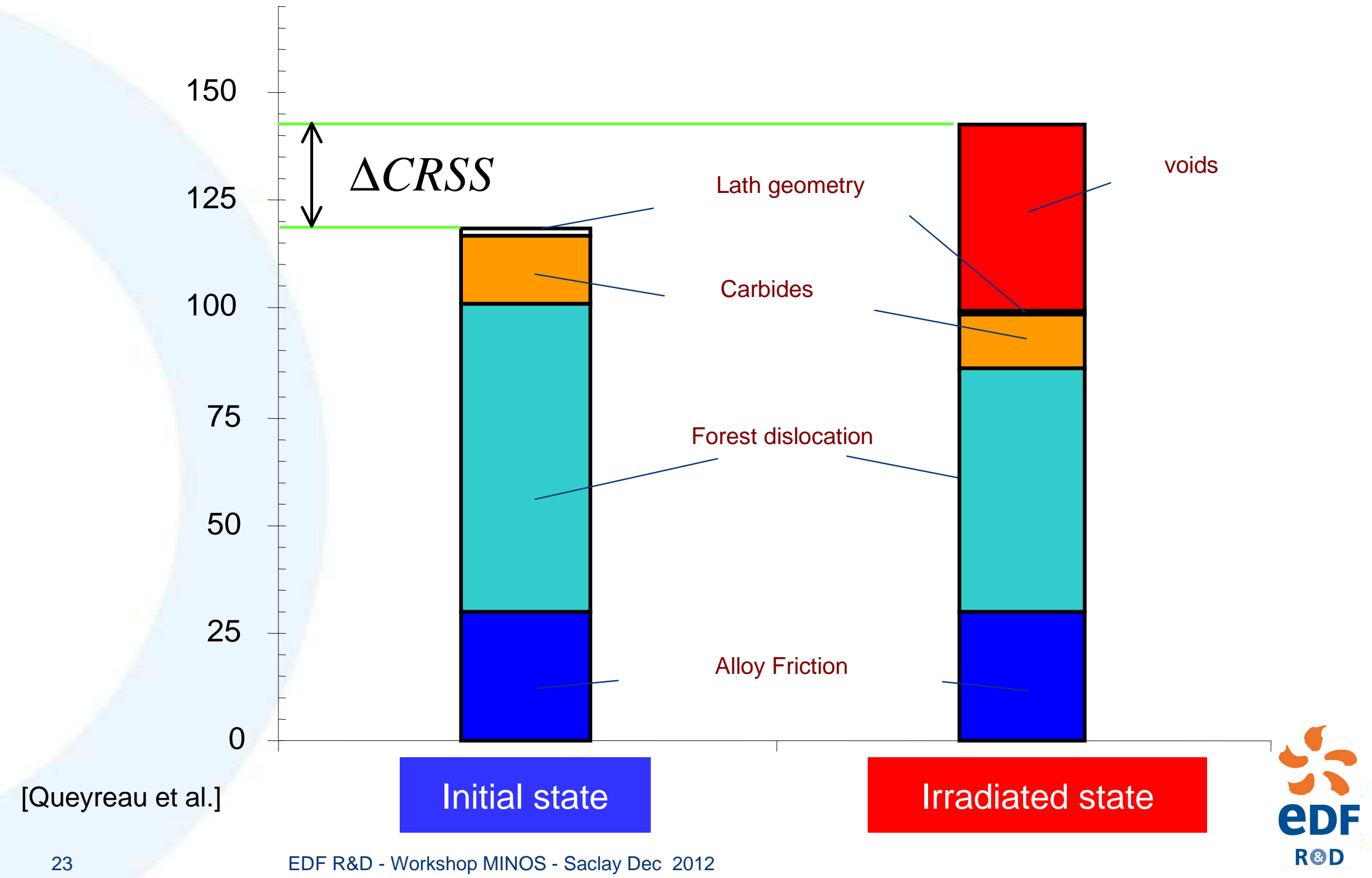




\section{Integration: RPV \& INTERN plateforms}

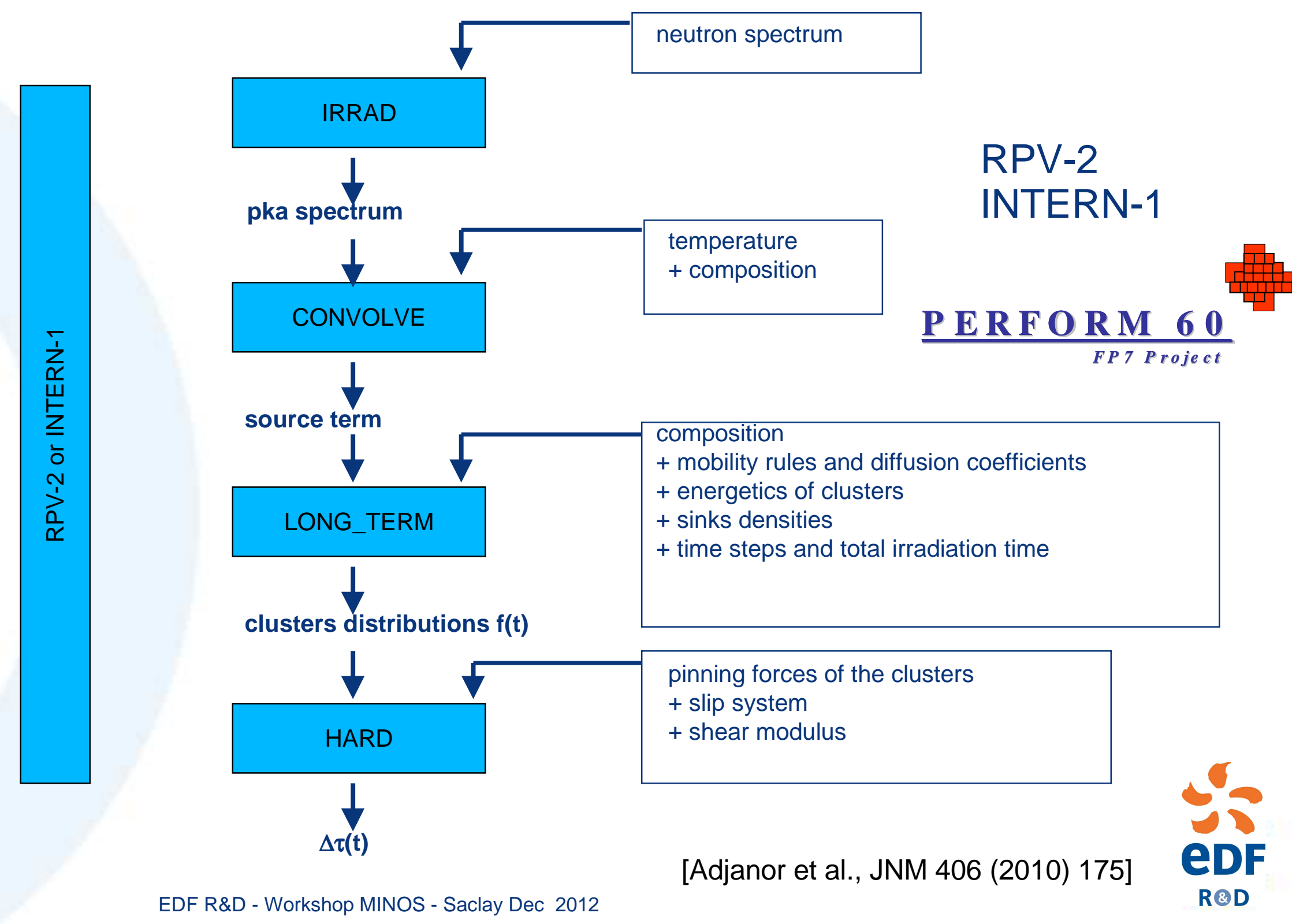




\section{Dislocation dynamics cristalline law of Fe alloys (at low temperature)}

DD velocity of scew dislo $v_{\text {screw }}(\tau, T)=\frac{v_{D} b^{2}}{l_{c}^{2}} l_{s c}^{s} \exp \left(-\frac{\Delta G\left(\tau_{a p p}\right)}{k T}\right)$
Orowan Law for plastic flow

$$
\dot{\gamma}_{s}=b \rho_{s c}^{s} v_{s c}^{s}(\tau, T)+b \rho_{e d}^{s} v_{e d}^{s}(\tau, T)
$$

[Naamane, Monnet, Devincre]
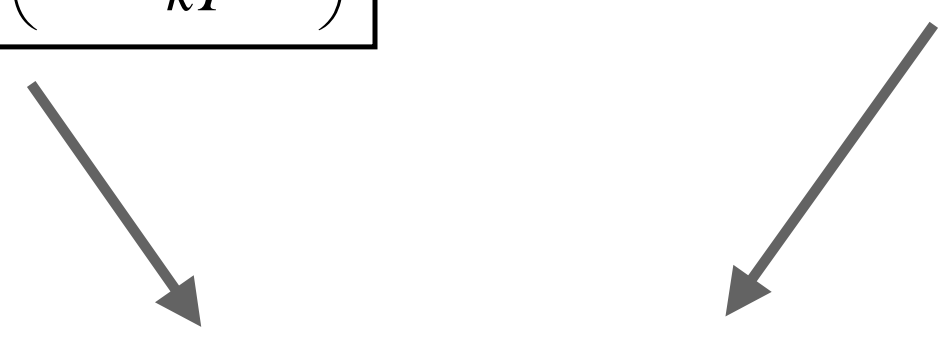

$$
\dot{\gamma}_{s}=4 \rho_{s c}^{s} \frac{v_{D} b^{3}}{l_{c}^{2}} l_{s c}^{s} \exp \left(-\frac{\Delta G_{o}}{k T}\right) \sinh \left(\frac{\Delta G_{o}}{k T} \sqrt{\frac{\tau_{e f f}^{s}}{\tau_{o}}}\right)
$$

Dislocation density evolution law

$$
\dot{\rho}_{s}=\frac{\left|\dot{\gamma}_{s}\right|}{b}\left(\frac{\sqrt{\sum a_{s u} \rho_{u}}}{K}-g_{c} \rho_{s}\right)
$$

[Monnet, Vincent, Mécanique \& Industries 12, 193-198 (2011)] EDF 


\section{Finite elements simulations: stress strain curves}
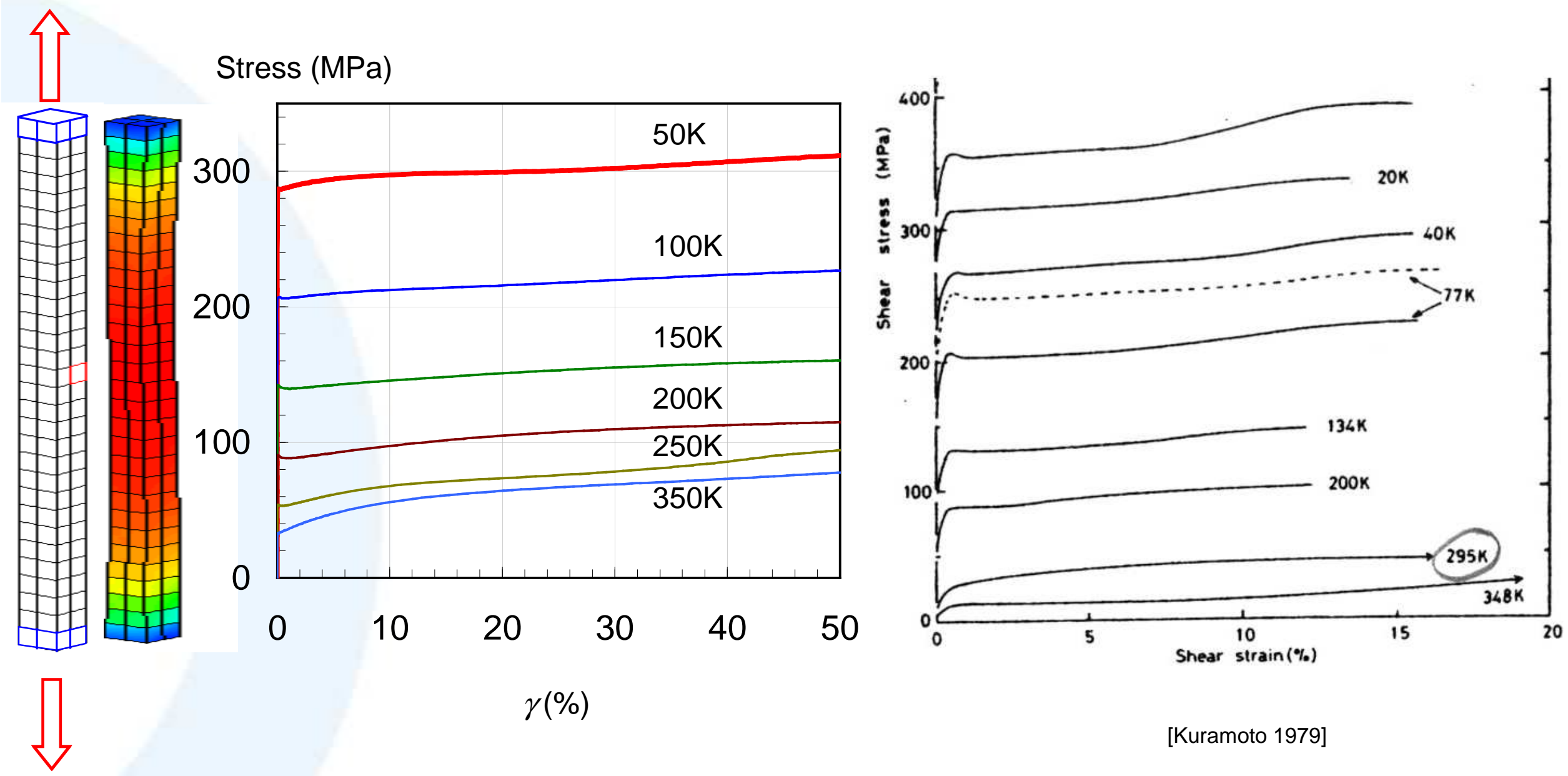

[Monnet, Vincent, Mécanique \& Industries 12, 193-198 (2011)] EDF 


\section{Material Multiscale Modeling Challenge}

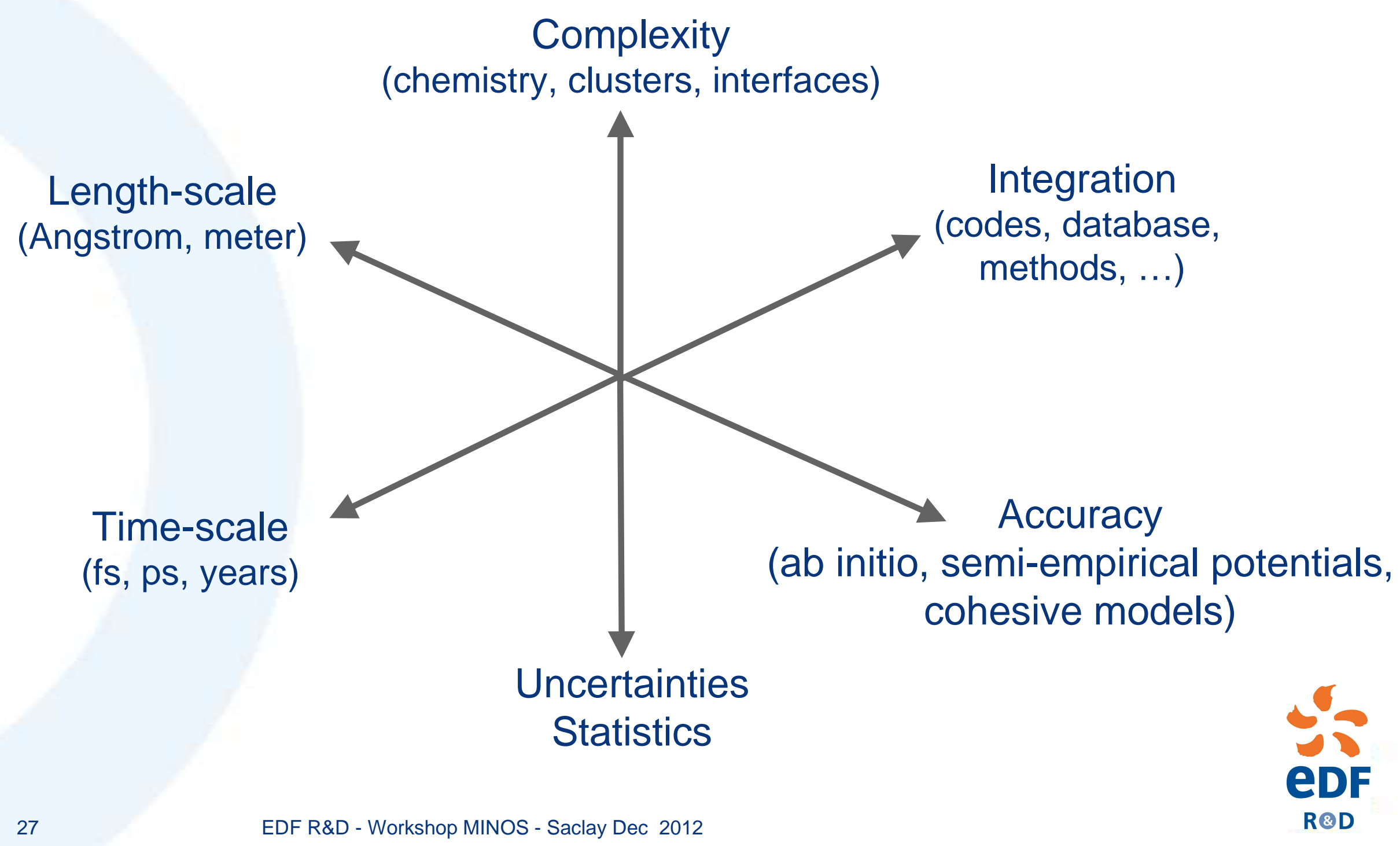




\section{Conclusions \& perspectives}

- A multi-scale modelling approach is developed for more than 10 years (e.g. through internal \& EURATOM European project SIRENA, PERFECT \& PERFORM60, GETMAT): RPV \& INTERN plateform.

- Improvement of the knowledge elementary properties allow to better predict material evolution.

- Some important progress thanks to the use of HPC machines.

- The prediction of the evolution of the mechanical properties requires to know the plasticity of the materials.

( ) The prediction of the evolution of the irradiated microstructure requires as input physical parameters the properties of each point defect clusters (mobility and stability).

- The properties of each point defect clusters (size, configurations, chemistry) need to be defined by ab initio calculations. Represent numerous configurations / mechanisms to be investigated. 